\title{
On Black-Box Ring Extraction and Integer Factorization ${ }^{\star}$
}

\author{
Kristina Altmann, Tibor Jager, and Andy Rupp \\ Horst Görtz Institute for IT-Security \\ Ruhr-University Bochum \\ \{kristina.altmann, tibor.jager\}@nds.rub.de, arupp@crypto.rub.de
}

\begin{abstract}
The black-box extraction problem over rings has (at least) two important interpretations in cryptography: An efficient algorithm for this problem implies (i) the equivalence of computing discrete logarithms and solving the Diffie-Hellman problem and (ii) the in-existence of secure ring-homomorphic encryption schemes.

In the special case of a finite field, Boneh/Lipton [BL96] and Maurer/Raub [MR07] show that there exist algorithms solving the black-box extraction problem in subexponential time. It is unknown whether there exist more efficient algorithms.

In this work we consider the black-box extraction problem over finite rings of characteristic $n$, where $n$ has at least two different prime factors. We provide a polynomial-time reduction from factoring $n$ to the black-box extraction problem for a large class of finite commutative unitary rings. Under the factoring assumption, this implies the in-existence of certain efficient generic reductions from computing discrete logarithms to the Diffie-Hellman problem on the one side, and might be an indicator that secure ring-homomorphic encryption schemes exist on the other side.
\end{abstract}

\section{Introduction}

Informally speaking, the black-box extraction problem over an algebraic structure $A$ (like a group, ring, or a field) can be described as follows: Given an explicit representation of $A$ (e.g., the cyclic group $\left(\mathbb{Z}_{n},+\right)$ with the canonical binary representation of elements) as well as access to a black-box resembling the structure of $A$ and hiding an element $x \in A$, the challenge is to recover $x$ in the given explicit representation. Algorithms that work on the black-box representation of an algebraic structure, and thus on any concrete representation, are called generic or black-box algorithms.

The black-box extraction problem has been studied in various variants and contexts, e.g., see [Nec94,Sho97,Mau05,BL96,MR07]. The case where the algebraic structure is a cyclic group (with given representation $\left(\mathbb{Z}_{n},+\right)$ ), and the extraction problem is better known as the discrete logarithm problem, was considered by Nechaev [Nec94] and Shoup [Sho97]. They showed that the expected running time of any generic algorithm for this problem is $\Omega(\sqrt{p})$, where $p$ is the largest prime factor of the group order $n$. Here, the integer $n$ as well as its factorization is assumed to be publicly known.

Boneh and Lipton [BL96] considered the black-box extraction problem over prime fields $\mathbb{F}_{p}$. Based on a result due to Maurer [Mau94] they developed an algorithm solving the problem in subexponential time (in $\log p$ ). Maurer and Raub [MR07] augmented this result to finite extension fields $\mathbb{F}_{p^{k}}$ by providing an efficient reduction from the black-box extraction problem over $\mathbb{F}_{p^{k}}$ to the black-box extraction problem over $\mathbb{F}_{p}$. Currently it is unknown whether there exist more efficient algorithms for black-box extraction over fields.

In this paper, we address the case where the underlying algebraic structure is a finite commutative ring with unity. The characteristic $n$ of the considered rings is the product of at least two different primes, thereby excluding the special case of a finite field. We provide an efficient reduction from computing a non-trivial factor of $n$ to the black-box extraction problem for virtually any such

\footnotetext{
* This is an extended version of the paper with the same title that appeared in the proceedings of ICALP 2008.
} 
ring where computations (i.e., applying the ring operations + and $\cdot$, equality tests and random sampling of elements) can be done efficiently.

The black-box extraction problem over fields/rings has at least two important applications in cryptography. For $\left(\mathbb{Z}_{n},+, \cdot\right)$ it can be interpreted as the problem of solving the discrete logarithm problem given access to an oracle for the Diffie-Hellman problem: $\left(\mathbb{Z}_{n},+\right)$ forms a cyclic additive group. The black-box provides access to the common operations on this group as well as to the additional operation ".". This extra operation can be interpreted as an oracle solving the DiffieHellman problem in the group $\left(\mathbb{Z}_{n},+\right)$. Hence, an efficient algorithm for the black-box extraction problem over $\left(\mathbb{Z}_{n},+, \cdot\right)$ would correspond to an efficient generic reduction from computing discrete logarithms to solving the Diffie-Hellman problem over cyclic groups of order $n$. Such reductions are known for groups where the group order is prime and meets certain properties [dB88], or if certain side information, depending on the respective group, is given [Mau94]. It is also known that no efficient generic reduction exists for groups with orders containing a large multiple prime factor [MW98]. Bach [Bac84] provided a reduction from factoring $n$ to computing discrete logarithms modulo $n$, i.e., in the multiplicative group $\mathbb{Z}_{n}^{*}$ of order $\phi(n)$, where $\phi(\cdot)$ denotes Euler's totient function.

Furthermore, the analysis of the black-box extraction problem sheds light on the existence of secure ring/field-homomorphic encryption schemes. Consider an encryption function enc : $K \times P \rightarrow$ $C$, where $K, P$ and $C$ denotes the key, plaintext and ciphertext space, respectively. Moreover, assume that $P$ and $C$ exhibit an algebraic structure with respect to certain operations. If for any $k \in K$ the function enc $_{k}:=\operatorname{enc}(k, \cdot)$ is a homomorphism from $P$ to $C$, the corresponding encryption scheme is said to be homomorphic. For instance, unpadded RSA is group-homomorphic, since the functions $\operatorname{enc}_{e}: \mathbb{Z}_{n} \rightarrow \mathbb{Z}_{n}, \operatorname{enc}_{e}(a):=a^{e}$ satisfy

$$
\operatorname{enc}_{e}(a \cdot b)=(a \cdot b)^{e}=a^{e} \cdot b^{e}=\operatorname{enc}_{e}(a) \cdot \operatorname{enc}_{e}(b),
$$

where "." denotes the multiplication modulo the RSA modulus $n$. Further well-known examples of group-homomorphic encryption schemes are native ElGamal [ElG85] and the Paillier cryptosystem [Pai99].

A natural question arising in this context is whether there exist secure ring-homomorphic encryption schemes, that is, schemes where $P$ and $C$ exhibit a ring structure, and enc ${ }_{k}$ is a ringhomomorphism. An efficient algorithm for the black-box extraction problem over the $\operatorname{ring} P$ would imply the inexistence of secure ring-homomorphic encryption schemes over $P$ : The black-box can be considered as an idealization of the encryption functions enc $c_{k}$ and the problem of recovering the explicit representation of $x$ as the problem of inverting enc $_{k}$. Note that since the black-box representation enables equality checking, also the class of considered encryption schemes allows for checking the equality of encrypted plaintexts. The results by Boneh and Lipton [BL96] and Maurer and Raub [MR07] imply that for the special case of a finite field any such scheme can be broken in subexponential time.

\subsection{Our Contribution}

In this work we consider the black-box extraction problem over finite commutative rings with unity whose characteristic $n$ is the product of at least two different primes. To the best of our knowledge, this case has not been treated in the literature yet. We present an efficient reduction from finding a non-trivial factor of $n$ to the black-box extraction problem over virtually any ring $R$ where computation is efficient. To this end, we extend a technique due to Leander and Rupp [LR06] which was originally used to prove the equivalence of breaking RSA and factoring regarding generic ring algorithms. 
We first provide a reduction for the case $R=\mathbb{Z}_{n}$. This case is especially interesting since Boneh and Lipton pointed out that their subexponential time black-box extraction algorithm for finite fields can be extended to finite rings $\mathbb{Z}_{n}$ if $n$ is square-free, requiring that the factorization of $n$ is known. Our result implies that there are no better algorithms than those that factorize $n$. Moreover, under the assumption that factoring $n$ is hard, this implies the in-existence of efficient generic reductions from computing discrete logarithms to solving the Diffie-Hellman problem in cyclic groups of order $n$. Note that in contrast to Bach [Bac84] presenting a reduction from factoring $n$ to computing discrete logarithms in the group $\mathbb{Z}_{n}^{*}$ of hidden order $\phi(n)$, we consider generic reductions in groups of known order $n$.

We extend our reduction to more general rings $R$ which are either given by a polynomial representation or a basis representation. More precisely, in the first case we consider rings of the form

$$
R=\mathbb{Z}_{n}\left[X_{1}, \ldots, X_{t}\right] / J
$$

where $t \geq 0$ and $J$ is an ideal in $\mathbb{Z}_{n}\left[X_{1}, \ldots, X_{t}\right]$ for which a Gröbner basis is known. In the second case, the ring $R$ is given by a tuple

$$
\left(n_{1}, \ldots, n_{t},\left(c_{i, j, k}\right)_{1 \leq i, j, k \leq t}\right)
$$

where $n_{1}, \ldots, n_{t}$ are the element orders of an additive basis of $(R,+)$ and the $c_{i, j, k}$ are integers describing the multiplicative relations of these basis elements. Finally, the reduction naturally extends to product rings

$$
R=R_{1} \times \ldots \times R_{\ell}
$$

where at least one component $R_{i}$ complies to one of the above forms.

We conclude that our results cover virtually any ring of cryptographic interest since choosing one of the above representations (currently) seems to be the only way that allows efficient computation in a finite commutative unitary ring without immediately revealing a factor of the ring characteristic.

Regarding secure homomorphic encryption our result has another interesting consequence: Boneh/Lipton [BL96] and Maurer/Raub [MR07] show that any field-homomorphic encryption scheme can be broken in subexponential time. It is an open question whether there exist more efficient generic algorithms. For a large class of rings we can negate this question, assuming that factoring the ring characteristic cannot be done better than in subexponential time. This might be seen as an indicator for the existence of secure ring-homomorphic encryption schemes.

\section{The Black-Box Ring Extraction Problem}

Informally speaking, black-box ring algorithms are the class of algorithms that operate on the structure of an algebraic ring without exploiting specific properties of the representation of ring elements. We adopt Shoup's generic group model [Sho97] to formalize the notion of black-box ring algorithms:

Let $(R,+, \cdot)$ be a finite commutative unitary ring and $S \subset\{0,1\}^{\left[\log _{2}(|R|)\right\rceil}$ be a set of bit strings of cardinality $|R|$. Let

$$
\sigma: R \rightarrow S
$$

be a bijective encoding function which assigns ring elements to bit strings, chosen at random among all possible bijections. A black-box ring algorithm is an algorithm that takes as input an encoding list $\left(\sigma\left(r_{1}\right), \ldots, \sigma\left(r_{k}\right)\right)$, where $r_{i} \in R$. Note that depending on the particular problem the algorithm might take some additional data as input, such as the characteristic of $R$, for example. In order to 
be able to perform the ring operations on randomly encoded elements, the algorithm may query a black-box ring oracle $\mathcal{O}$. The oracle takes two indices $i, j$ into the encoding list and a symbol $\circ \in\{+,-, \cdot\}$ as input, computes $\sigma\left(r_{i} \circ r_{j}\right)$ and appends this bit string to the encoding list (to which the algorithm always has access).

We capture the notion of a black-box ring representation by the following definition:

Definition 1 (Black-Box Ring Representation). Let $(R,+, \cdot)$ be a finite ring. We call the tuple $(\sigma, \mathcal{O})$ consisting of a randomly chosen encoding function $\sigma: R \rightarrow S$, and a corresponding black-box ring oracle $\mathcal{O}$ a black-box ring representation for $R$ and denote it by $R^{\sigma}$.

For short, we sometimes call $R^{\sigma}$ a black box ring (meaning that we consider a ring exhibiting the structure of $R$ but whose elements are encoded by random bit strings). As an abuse of notation we occasionally write $\sigma(x) \in R^{\sigma}$ meaning that the unique encoding $\sigma(x)$ of an element $x \in R$ is given. Moreover, when we say in the following that an algorithm $\mathcal{A}$ performs operations on the black-box ring $R^{\sigma}$, we mean that $\mathcal{A}$ interacts with the black-box ring oracle as described above.

Having formalized the notion of a black-box ring, we can define the black-box ring extraction (BBRE) problem:

Definition 2 (BBRE Problem). Let $R$ be an explicitly given finite commutative ring with unity 1 and known characteristic $n$. Furthermore, let $B:=\left\{r_{1}, \ldots, r_{t}\right\}$ be an (explicitly given) generating set of $R$. The black-box ring extraction (BBRE) problem for $R$ is the task of computing $x \in R$, where $x$ is chosen uniformly random from $R$, given $\sigma(x), \sigma(1), \sigma\left(r_{1}\right), \ldots, \sigma\left(r_{t}\right) \in R^{\sigma}$.

In the following we consider the BBRE problem over different rings $R$ and relate the hardness of this problem to the hardness of integer factorization (IF) problem, more precisely, to problem of finding a factor of $n$.

\section{The Relation between BBRE and IF for $\mathbb{Z}_{n}$}

In this section we consider the BBRE problem for the ring $\mathbb{Z}_{n}$, where $n$ has at least two different prime factors. We provide a reduction from factoring $n$ to the BBRE problem in the following sense: If there exists an efficient algorithm solving the BBRE problem for $\mathbb{Z}_{n}$ with non-negligible success probability, then there exists an efficient algorithm finding a factor of $n$ with non-negligible probability.

Theorem 1. Let $R:=\mathbb{Z}_{n}$ for some integer $n$ having at least two different prime factors. Let $\mathcal{A}$ be an algorithm for the BBRE problem that performs at most $m \leq n$ operations on $R^{\sigma}$. Assume that $\mathcal{A}$ solves the BBRE problem with probability $\epsilon$. Then there is an algorithm $\mathcal{B}$ having white-box access to $\mathcal{A}$ that finds a factor of $n$ with probability at least

$$
\frac{\epsilon-\frac{1}{n}}{m^{2}+3 m+2}
$$

by running $\mathcal{A}$ once and performing an additional amount of $O\left(\mathrm{~m}^{2}\right)$ random choices and $O\left(\mathrm{~m}^{3}\right)$ operations on $R$ as well as $O\left(m^{2}\right)$ gcd computations on $\log _{2}(n)$-bit numbers.

Remark 1. Assume we have a BBRE algorithm $\mathcal{A}$ that works for all rings $R=\mathbb{Z}_{n}$ where $n$ consists of at least two different prime factors. Then algorithm $\mathcal{B}$ can be used to factor a given integer $n$ completely. This is done by first running $\mathcal{B}$ on $n$, i.e., $\mathcal{B}$ runs $\mathcal{A}$ on an instance of the BBRE problem over $\mathbb{Z}_{n}$ and performs some additional operations, resulting in a factor $d$ of $n$ with a certain probability. If $n / d$ is not a prime power, which can easily be determined, we can run $\mathcal{B}$ on $n / d$ and so on. If $\mathcal{A}$ is efficient and solves the BBRE problem with non-negligible probability, then the same holds for the resulting factoring algorithm. 
Proof Outline. In a nutshell, our proof works as follows: We replace the original black-box ring oracle $\mathcal{O}$ with an oracle $\mathcal{O}_{\text {sim }}$ that simulates $\mathcal{O}$ without using the knowledge of the secret $x$. We call this setting the simulation game. Then we show that the behavior of $\mathcal{O}_{\text {sim }}$ is perfectly indistinguishable from $\mathcal{O}$ unless a certain simulation failure $\mathbf{F}$ occurs. Denoting the success event of $\mathcal{A}$ when interacting with $\mathcal{O}$ and $\mathcal{O}_{\text {sim }}$ by $\mathbf{S}$ and $\mathbf{S}_{\text {sim }}$, respectively, it immediately follows that $\epsilon=\operatorname{Pr}[\mathbf{S}]$ is upper bound by $\operatorname{Pr}\left[\mathbf{S}_{\text {sim }}\right]+\operatorname{Pr}[\mathbf{F}]$. In other words, the probability $\operatorname{Pr}[\mathbf{F}]$ of a failure is at least $\epsilon-\operatorname{Pr}\left[\mathbf{S}_{\text {sim }}\right]$. Showing that $\operatorname{Pr}[\mathbf{F}]$ (multiplied by a certain prefactor) is in turn a lower bound on the the probability of revealing a divisor of $n$ completes our proof.

\subsection{Detailed Proof of Theorem 1}

Before introducing the actual simulation oracle, as announced in the proof outline, let us first define a slightly modified but equivalent version of the original black-box ring oracle $\mathcal{O}$ : Instead of using the ring $R=\mathbb{Z}_{n}$ for the internal representation of ring elements, these elements are represented by polynomials in the variable $X$ over $R$ which are evaluated with $x$ each time the encoding of a newly computed element must be determined.

Definition 3 (An Equivalent Oracle). The oracle $\mathcal{O}^{\prime}$ has an input and an output port as well as a random tape and performs computations as follows.

Input. As input $\mathcal{O}^{\prime}$ receives the modulus $n$ and an element $x \in_{U} R$.

Internal State. As internal state $\mathcal{O}^{\prime}$ maintains two lists $L \subset R[X]$ and $E \subset S_{n}$. For an index $i$ let $L_{i}$ and $E_{i}$ denote the $i$-th element of $L$ and $E$, respectively.

Encoding of Elements. Each time a polynomial $P$ should be appended to the list $L$ the following computation is triggered to determine the encoding of $P(x): \mathcal{O}^{\prime}$ checks if there exists any index $1 \leq i \leq|L|$ such that

$$
\left(P-L_{i}\right)(x) \equiv 0 \bmod n .
$$

If this equation holds for some $i$, then the respective encoding $E_{i}$ is appended to $E$ again. Otherwise the oracle chooses a new encoding $s \in_{U} S \backslash E$ and appends it to $E$.

The computation of $\mathcal{O}^{\prime}$ starts with an initialization phase, which is run once, followed by the execution of the query-handling phase:

Initialization. The list $L$ is initialized with the polynomials $1, X$ and the list $E$ is initialized with corresponding encodings.

Query-handling. Upon receiving a query $\left(\circ, i_{1}, i_{2}\right)$ on its input tape, where $\circ \in\{+,-, \cdot\}$ identifies an operation and $i_{1}, i_{2}$ are indices identifying the list elements the operation should be applied to, $\mathcal{O}^{\prime}$ appends the polynomial $P:=L_{i_{1}} \circ L_{i_{2}}$ to $L$ and the corresponding encoding to $E$.

We say that an algorithm is successful in this game iff it outputs $x$ and denote this event by $\mathbf{S}$.

A Simulation Game. Now we replace $\mathcal{O}^{\prime}$ by a simulation oracle $\mathcal{O}_{\text {sim }}$. The simulation oracle is defined exactly like $\mathcal{O}^{\prime}$ except that it determines the encodings of elements in a different way in order to be independent of the secret $x$.

Each time a polynomial $P$ is appended to the end of list $L$ (during initialization or queryhandling), $\mathcal{O}_{\text {sim }}$ does the following: Let $L_{j}=P$ denote the last entry of the updated list. Then for each $1 \leq i<j$ the simulation oracle chooses a new element $x_{i, j} \in R$ uniformly at random and checks whether the equation

$$
\left(L_{i}-L_{j}\right)\left(x_{i, j}\right) \equiv 0 \bmod n
$$

holds. If it is not satisfied for any $i$, the oracle chooses a new encoding $s \in_{U} S \backslash E$ and appends it to $E$. Otherwise, for the first $i$ the equation is satisfied, the corresponding encoding $E_{i}$ is appended 
to $E$ again (i.e., $E_{j}=E_{i}$ ). The algorithm is successful in the simulation game if it outputs the element $x$ (given as input to $\mathcal{O}_{\text {sim }}$ ). We denote this event by $\mathbf{S}_{\text {sim }}$.

Note that due to the modification of the element encoding procedure, it is now possible that both an element $L_{i}(x)$ is assigned to two or more different encodings and that different elements are assigned to the same encoding. In these cases the behavior of $\mathcal{O}_{\text {sim }}$ differs from that of $\mathcal{O}^{\prime}$, what may allow to distinguish between the oracles. In the case of a differing behavior the following failure event $\mathbf{F}$ occurred: There exist $i, j \in\{1, \ldots,|L|\}$ satisfying the equation

$$
\left(L_{i}-L_{j}\right)(x) \equiv 0 \bmod n \text { and }\left(L_{i}-L_{j}\right)\left(x_{i, j}\right) \not \equiv 0 \bmod n
$$

or the equation

$$
\left(L_{i}-L_{j}\right)(x) \not \equiv 0 \bmod n \text { and }\left(L_{i}-L_{j}\right)\left(x_{i, j}\right) \equiv 0 \bmod n .
$$

Remark 2. There is a technical subtlety. If there is $i<j$ such that $\left(L_{i}-L_{j}\right)(x) \equiv 0 \bmod n$ but $\left(L_{i}-L_{j}\right)\left(x_{i, j}\right) \not \equiv 0 \bmod n$ then $\mathcal{O}_{\text {sim }}$ does not necessarily determine different encodings for $L_{j}(x)$. There may be some $i<i^{\prime}<j$ such that $\left(L_{i^{\prime}}-L_{j}\right)(x) \equiv 0 \bmod n$ and $\left(L_{i^{\prime}}-L_{j}\right)\left(x_{i^{\prime}, j}\right) \equiv 0 \bmod n$ and $E_{i}=E_{i^{\prime}}$. So the simulation failure event as defined by us is just a necessary but not a sufficient condition for discriminative behavior of $\mathcal{O}^{\prime}$ and $\mathcal{O}_{\text {sim }}$.

It is important to observe that the original game and the simulation game proceed identically unless $\mathbf{F}$ occurs: To this end consider the algorithm $\mathcal{A}$ as deterministic Turing machine with identical input and random tape in both games. Also, consider the oracles $\mathcal{O}^{\prime}$ and $\mathcal{O}_{\text {sim }}$ as deterministic Turing machines receiving the same inputs and random tapes. ${ }^{1}$ Assuming that $\mathbf{F}$ does not occur, the algorithm receives the same sequence of encodings and thus issues the same sequence of queries in both games. Furthermore, it outputs the same element in the end of both games and thus wins the simulation game if and only if it wins the original game. Hence, we have the following relation between the considered events

$$
\mathbf{S} \wedge \neg \mathbf{F} \Longleftrightarrow \mathbf{S}_{\text {sim }} \wedge \neg \mathbf{F} .
$$

We can obtain an upper bound on $\operatorname{Pr}[\mathbf{S}]$ by deriving upper bounds on $\operatorname{Pr}\left[\mathbf{S}_{\text {sim }}\right]$ and $\operatorname{Pr}[\mathbf{F}]$ and applying the Difference Lemma (Lemma 1).

Lemma 1 (Difference Lemma [Sho04]). Let $\mathbf{E}_{1}, \mathbf{E}_{2}$, and $\mathbf{E}_{3}$ be events over the same probability space. If $\mathbf{E}_{1} \wedge \neg \mathbf{E}_{3} \Longleftrightarrow \mathbf{E}_{2} \wedge \neg \mathbf{E}_{3}$, then it holds that

$$
\left|\operatorname{Pr}\left[\mathbf{E}_{1}\right]-\operatorname{Pr}\left[\mathbf{E}_{2}\right]\right| \leq \operatorname{Pr}\left[\mathbf{E}_{3}\right] .
$$

Bounding the Probability of Success in the Simulation Game. Since all computations are independent of the uniformly random element $x \in R$, the algorithm $\mathcal{A}$ can only guess $x$ :

$$
\operatorname{Pr}\left[\mathbf{S}_{\text {sim }}\right] \leq \frac{1}{|R|}=\frac{1}{n}
$$

Bounding the Probability of a Simulation Failure. Let $\mathfrak{D}=\left\{L_{i}-L_{j}|1 \leq i<j \leq| L \mid\right\}$ denote the set of all non-trivial differences of polynomials in $L$ after a run of $\mathcal{A}$. In the following we show how the probability that a polynomial $\Delta \in \mathfrak{D}$ causes a simulation failure is related to the probability of revealing a factor of $n$ by simply evaluating $\Delta$ with a uniformly random element from $R$.

\footnotetext{
${ }^{1}$ To be precise here, we actually should have defined $\mathcal{O}^{\prime}$ to perform exactly the same random choices as $\mathcal{O}_{\text {sim }}$ (i.e., letting $\mathcal{O}^{\prime}$ also choose the elements $x_{i, j}$ but without using them).
} 
For fixed $\Delta \in \mathfrak{D}$ let $\mathbf{F}_{\Delta}$ denote the event that $\Delta$ causes a simulation failure as defined by Equations (1) and (2). Furthermore, let $\mathbf{D}_{\Delta}$ denote the event that $\operatorname{gcd}(n, \Delta(a)) \notin\{1, n\}$ when choosing an element $a$ uniformly at random from $R$.

Now, we are going to express the probabilities of both events using the same terms. Let $n=$ $\prod_{i=1}^{k} p_{i}^{e_{i}}$ be the prime factor decomposition of $n$. Hence, $R$ is isomorphic to $\mathbb{Z}_{p_{1}^{e_{1}}} \times \ldots \times \mathbb{Z}_{p_{k} e_{k}}$ by the Chinese Remainder Theorem. Then we can write

$$
\begin{aligned}
\operatorname{Pr}_{a \in_{U} R}[\Delta(a) \equiv 0 \bmod n] & =\operatorname{Pr}_{a \in_{U} R}\left[\left(\Delta(a) \equiv 0 \bmod p_{1}^{e_{1}}\right) \wedge \ldots \wedge\left(\Delta(a) \equiv 0 \bmod p_{k}^{e_{k}}\right)\right] \\
& =\prod_{i=1}^{k} \operatorname{Pr}_{a \in_{U} R}\left[\Delta(a) \equiv 0 \bmod p_{i}^{e_{i}}\right] \\
& =\prod_{i=1}^{k} \nu_{i}
\end{aligned}
$$

where $\nu_{i}:=\frac{\left|\left\{a \in R \mid \Delta(a) \equiv 0 \bmod p_{i}^{e_{i}}\right\}\right|}{|R|}$. Note that the second line of the above equation follows from the fact that the events defined by the predicates $\Delta(a) \equiv 0 \bmod p_{i}^{e_{i}}$ are mutually independent. Using Equation (3) we can express the probability of $\mathbf{F}_{\Delta}$ by

$$
\begin{aligned}
\operatorname{Pr}\left[\mathbf{F}_{\Delta}\right] & =\operatorname{Pr}_{a \in_{U} R}[\Delta(a) \equiv 0 \bmod n]\left(1-\operatorname{Pr}_{a \in_{U} R}[\Delta(a) \equiv 0 \bmod n]\right) \\
& +\operatorname{Pr}_{a \in_{U} R}[\Delta(a) \equiv 0 \bmod n]\left(1-\operatorname{Pr}_{a \in_{U} R}[\Delta(a) \equiv 0 \bmod n]\right) \\
& =2\left(1-\prod_{i=1}^{k} \nu_{i}\right)\left(\prod_{i=1}^{k} \nu_{i}\right) .
\end{aligned}
$$

Similarly, we can write the probability of $\mathbf{D}_{\Delta}$ as

$$
\begin{aligned}
\operatorname{Pr}\left[\mathbf{D}_{\Delta}\right] & =1-\operatorname{Pr}_{a \in U}[\Delta(a) \equiv 0 \bmod n]-\operatorname{Pr}_{a \in U}\left[\left(\Delta(a) \not \equiv 0 \bmod p_{1}^{e_{1}}\right) \wedge \ldots \wedge\left(\Delta(a) \not \equiv 0 \bmod p_{k}^{e_{k}}\right)\right] \\
& =1-\operatorname{Pr}_{a \in_{U} R}[\Delta(a) \equiv 0 \bmod n]-\prod_{i=1}^{k} \operatorname{Pr}_{a \in U}\left[\Delta(a) \not \equiv 0 \bmod p_{i}^{e_{i}}\right] \\
& =1-\prod_{i=1}^{k} \nu_{i}-\prod_{i=1}^{k}\left(1-\nu_{i}\right)
\end{aligned}
$$

Now, the key observation is that we have the following relation between the probabilities of the events $\mathbf{F}_{\Delta}$ and $\mathbf{D}_{\Delta}$ :

Lemma 2. $\forall \Delta \in \mathfrak{D}: 2 \operatorname{Pr}\left[\mathbf{D}_{\Delta}\right] \geq \operatorname{Pr}\left[\mathbf{F}_{\Delta}\right]$

Proof. We have

$$
\begin{aligned}
& 2 \operatorname{Pr}\left[\mathbf{D}_{\Delta}\right]-\operatorname{Pr}\left[\mathbf{F}_{\Delta}\right]=2\left(1-2 \prod_{i=1}^{k} \nu_{i}-\prod_{i=1}^{k}\left(1-\nu_{i}\right)+\prod_{i=1}^{k} \nu_{i}^{2}\right) \geq 0 \\
& \Longleftrightarrow\left(1-\prod_{i=1}^{k} \nu_{i}\right)^{2} \geq \prod_{i=1}^{k}\left(1-\nu_{i}\right)
\end{aligned}
$$


It is easy to prove by induction over $k$ that the inequality

$$
\left(1-\prod_{i=1}^{k} \nu_{i}\right)^{k} \geq \prod_{i=1}^{k}\left(1-\nu_{i}\right)
$$

holds for all $k \geq 1$. From this our claim follows immediately since

$$
\left(1-\prod_{i=1}^{k} \nu_{i}\right)^{2} \geq\left(1-\prod_{i=1}^{k} \nu_{i}\right)^{k}
$$

holds for all $k \geq 2$.

The Factoring Algorithm. Based on the relation given by Lemma 2, we construct an efficient factoring algorithm. Consider an algorithm $\mathcal{B}$ that runs the BBRE algorithm $\mathcal{A}$ on an arbitrary instance of the BBRE problem over $\mathbb{Z}_{n}$. During this run it records the sequence of queries that $\mathcal{A}$ issues, i.e., it records the same list $L$ of polynomials as the black-box ring oracle. Then for each $\Delta \in \mathfrak{D}$ the algorithm $\mathcal{B}$ chooses a new random element $a \in \mathbb{Z}_{n}$ (like the oracle is doing), and computes $\operatorname{gcd}(n, \Delta(a))$. There are at most $(m+2)(m+1) / 2$ such polynomials and each of them can be evaluated using at most $m+1$ ring operations (since it is given as a straight-line program of length at most $m$ ). Thus, $\mathcal{B}$ chooses $O\left(\mathrm{~m}^{2}\right)$ random elements and performs $O\left(\mathrm{~m}^{3}\right)$ operations on $R$ as well as $O\left(m^{2}\right)$ gcd computations on $\log _{2}(n)$-bit numbers. Let the event that at least one of these gcd computations yields a non-trivial factor of $n$ be denoted by $\mathbf{D}$. Then clearly we have

$$
\operatorname{Pr}[\mathbf{D}] \geq \max _{\Delta \in \mathfrak{D}}\left(\operatorname{Pr}\left[\mathbf{D}_{\Delta}\right]\right) .
$$

From this we can derive the following lower bound on the the success probability of $\mathcal{B}$ in terms of the failure probability:

$$
\operatorname{Pr}[\mathbf{F}] \leq \sum_{\Delta \in \mathfrak{D}} \operatorname{Pr}\left[\mathbf{F}_{\Delta}\right] \leq 2 \sum_{\Delta \in \mathfrak{D}} \operatorname{Pr}\left[\mathbf{D}_{\Delta}\right] \leq(m+2)(m+1) \operatorname{Pr}[\mathbf{D}]
$$

To Summarize. Remember that $\epsilon=\operatorname{Pr}[\mathbf{S}]$ denotes the success probability of the BBRE algorithm $\mathcal{A}$. From Lemma 1 follows that

$$
\operatorname{Pr}[\mathbf{F}] \geq \operatorname{Pr}[\mathbf{S}]-\operatorname{Pr}\left[\mathbf{S}_{\text {sim }}\right] \geq \epsilon-\frac{1}{n} .
$$

Deploying the above lower bound on $\operatorname{Pr}[\mathbf{F}]$ in Equation (6) finally yields

$$
\operatorname{Pr}[\mathbf{D}] \geq \frac{\operatorname{Pr}[\mathbf{F}]}{(m+2)(m+1)} \geq \frac{\epsilon-\frac{1}{n}}{(m+2)(m+1)} .
$$

\section{Extending our Reduction to Multivariate Polynomial Rings}

In this section we are going to lift our reduction from the special case $R=\mathbb{Z}_{n}$ to the case

$$
R=\mathbb{Z}_{n}\left[X_{1}, \ldots, X_{t}\right] / J
$$

where $\mathbb{Z}_{n}\left[X_{1}, \ldots, X_{t}\right]$ denotes the ring of polynomials over $\mathbb{Z}_{n}$ in indeterminates $X_{1}, \ldots, X_{t}(t \geq 0)$ and $J$ is an ideal in this polynomial ring such that $R$ is finite. Note that any finite commutative ring with unity can be represented in this way: 
Lemma 3 (Polynomial Representation). Let $R$ be a finite commutative unitary ring of characteristic $n$. Then there is a number $t \leq \log _{2}|R|$ and a finitely generated ideal $J$ of $\mathbb{Z}_{n}\left[X_{1}, \ldots, X_{t}\right]$ such that $R \cong \mathbb{Z}_{n}\left[X_{1}, \ldots, X_{t}\right] / J$.

Proof. Let $M=\left\{m_{1}, \ldots m_{t}\right\} \subset R$ be a generating subset of $R$, i.e., $R=\langle M\rangle$. Consider the mapping $\phi: \mathbb{Z}_{n}\left[X_{1}, \ldots, X_{t}\right] \rightarrow R$ such that $1 \mapsto 1$ and $X_{i} \mapsto m_{i}$ for $1 \leq i \leq t$. Certainly, $\phi$ is a ring homomorphism implying that $J:=\operatorname{ker}(\phi)$ is an ideal of $\mathbb{Z}_{n}\left[X_{1}, \ldots, X_{t}\right]$. Applying the fundamental theorem on homomorphisms (e.g., see $\left[\operatorname{Lan02}\right.$, p.89]) yields that $\mathbb{Z}_{n}\left[X_{1}, \ldots, X_{t}\right] / J \cong R$.

Since $\mathbb{Z}_{n}$ trivially is a Noetherian ring it follows by Hilbert's basis theorem (e.g, see [Gri99, p.181]) that also the polynomial $\operatorname{ring} \mathbb{Z}_{n}\left[X_{1}, \ldots, X_{t}\right]$ is Noetherian. Thus, every ideal of $\mathbb{Z}_{n}\left[X_{1}, \ldots, X_{t}\right]$, especially $J$, is finitely generated.

By the fundamental theorem of finitely generated abelian groups (e.g., see [Gri99, p.48]) the additive group of a finite ring decomposes uniquely (up to order) into a direct product of cyclic groups. Observe that a group of cardinality $|R|$ decomposes into a product of at most $\log _{2}|R|$ groups. Hence, setting $M$ to be the set of generators of these subgroups of $(R,+)$, we see that a number of $t \leq \log _{2}|R|$ elements is sufficient to generate the entire ring.

We start by considering a useful decomposition of such rings similar to the CRT-decomposition for $\mathbb{Z}_{n}$. Next, we give some facts about Gröbner bases over these rings and their component rings. Finally, we use these results in a reduction proof which is similar to the one for $\mathbb{Z}_{n}$.

\subsection{A Prime-Power Decomposition for Multivariate Polynomial Rings}

It is a well-known fact that any finite commutative ring is uniquely (up to order) decomposable into a direct product of local rings [McD74, p.95]. However, since this decomposition does not meet our requirements, we devise another simple way of decomposing $R$ into a direct product of rings with prime-power characteristic, but not necessarily local rings.

Lemma 4. Let $R=\mathbb{Z}_{n}\left[X_{1}, \ldots, X_{t}\right] /\langle F\rangle$ and $n=\prod_{i=1}^{k} p_{i}^{e_{i}}$ be the prime factor decomposition of the characteristic $n$ of $R$. Then $R$ is decomposable into a direct product of rings

$$
R \cong R_{1} \times \ldots \times R_{k}
$$

where $R_{i}:=\mathbb{Z}_{p_{i} e_{i}}\left[X_{1}, \ldots, X_{t}\right] / J$.

Proof. Let $F$ be a set of polynomials generating the ideal $J$ in $\mathbb{Z}_{n}\left[X_{1}, \ldots, X_{t}\right]$. We denote this by $J=\langle F\rangle$. Note that the ring $R$ can equivalently be written as $\mathbb{Z}\left[X_{1}, \ldots, X_{t}\right] /\langle n, F\rangle$. For $1 \leq i \leq k$ let $J_{i}:=\left\langle p_{i}^{e_{i}}, F\right\rangle$ which is an ideal in $\mathbb{Z}\left[X_{1}, \ldots, X_{t}\right]$. Then for each $1 \leq i<j \leq k$ it holds that $J_{i}+J_{j}:=\left\{a+b \mid a \in J_{i}, b \in J_{j}\right\}=\mathbb{Z}\left[X_{1}, \ldots, X_{t}\right]$. Moreover, we have $\bigcap_{i=1}^{k} J_{i}=\langle n, F\rangle$. Thus, by the generalized Chinese Remainder Theorem [Gri99, p.184], we obtain the isomorphism

$$
\begin{aligned}
\mathbb{Z}_{n}\left[X_{1}, \ldots, X_{t}\right] /\langle F\rangle & \cong \mathbb{Z}\left[X_{1}, \ldots, X_{t}\right] /\langle n, F\rangle \\
& \cong \mathbb{Z}\left[X_{1}, \ldots, X_{t}\right] /\left\langle p_{1}^{e_{1}}, F\right\rangle \times \ldots \times \mathbb{Z}\left[X_{1}, \ldots, X_{t}\right] /\left\langle p_{k}^{e_{k}}, F\right\rangle \\
& \cong \mathbb{Z}_{p_{1}^{e_{1}}}\left[X_{1}, \ldots, X_{t}\right] / J \times \ldots \times \mathbb{Z}_{p_{k}^{e_{k}}}\left[X_{1}, \ldots, X_{t}\right] / J
\end{aligned}
$$

We call this way of decomposing $R$ the prime-power decomposition of $R$. Note that Lemma 4 holds for any finite commutative unitary ring since any such ring can be represented as polynomial ring:

Corollary 1. Let $R$ be a finite commutative unitary ring of characteristic $n=\prod_{i=1}^{k} p_{i}^{e_{i}}$. Then $R$ is isomorphic to a product of rings

$$
R_{1} \times \cdots \times R_{k}
$$

where $R_{i}$ has characteristic $p_{i}^{e_{i}}$. 


\subsection{Gröbner Bases for Polynomial Ideals over Rings}

Roughly speaking, a Gröbner basis $G$ is a generating set of an ideal $J$ in a multivariate polynomial ring exhibiting the special property that reduction of polynomials from $J$ modulo the set $G$ always yields the residue zero. This property is not satisfied for arbitrary ideal bases and enables effective computation in residue class rings modulo polynomial ideals in the first place. Gröbner bases were originally introduced by Buchberger [Buc65] for ideals $J$ in $K\left[X_{1}, \ldots, X_{t}\right.$ ] where the coefficient space $K$ is a field. Later this notion was generalized to the case where $K$ is a Noetherian ring such as $\mathbb{Z}_{n}$ (e.g., see [AL94, Chapter 4]).

Let us introduce some notation. A monomial or power product in indeterminates $X_{1}, \ldots, X_{t}$ is a product of the form $\mathcal{X}=X_{1}^{a_{1}} \ldots X_{t}^{a_{t}}$ for some $\left(a_{1}, \ldots, a_{t}\right) \in \mathbb{N}_{0}^{t}$. In the following let an arbitrary but admissible order $>$ on monomials be given. For instance, this could be the lexicographic order $>_{\text {lex }}$ defined as: $\mathcal{X}_{1}=X_{1}^{a_{1}} \cdots X_{t}^{a_{t}}>_{\text {lex }} \mathcal{X}_{2}=X_{1}^{b_{1}} \cdots X_{t}^{b_{t}}$ iff the leftmost non-zero entry of $\left(a_{1}-\right.$ $\left.b_{1}, \ldots, a_{t}-b_{t}\right)$ is positive.

Let $f \in \mathbb{Z}_{n}\left[X_{1}, \ldots, X_{t}\right]$ with $f \neq 0$. Then we can write $f$ as $f=c_{1} \mathcal{X}_{1}+\ldots+c_{s} \mathcal{X}_{s}$, where $c_{1}, \ldots, c_{s} \in \mathbb{Z}_{n} \backslash\{0\}$ and $\mathcal{X}_{1}>\ldots>\mathcal{X}_{s}$. The leading coefficient $\operatorname{lc}(f)$, the leading monomial $\operatorname{lp}(f)$, and the leading term $\operatorname{lt}(f)$ of $f$ with respect to $>$ are defined as $\operatorname{lc}(f):=a_{1}, \operatorname{lp}(f):=\mathcal{X}_{1}$, and $\operatorname{lt}(f):=a_{1} \mathcal{X}_{1}$, respectively.

Now, we are able to define the reduction of a polynomial modulo a set of polynomials. To this end, we adopt the respective definitions from [AL94, Chapter 4]. In the following we do not mention the fixed monomial ordering explicitly anymore.

Definition 4 (Polynomial Reduction). Let two polynomials $f$ and $h$ and a set of non-zero polynomials $F=\left\{f_{1}, \ldots, f_{s}\right\}$ in $\mathbb{Z}_{n}\left[X_{1}, \ldots, X_{t}\right]$ be given .

(a) We say that $f$ can be reduced to $h$ modulo $F$ in one step, denoted by $f \stackrel{F}{\longrightarrow} h$, if and only if $h=f-\left(c_{1} \mathcal{X}_{1} f_{1}+\ldots+c_{s} \mathcal{X}_{s} f_{s}\right)$ for $c_{1}, \ldots, c_{s} \in R$ and power products $\mathcal{X}_{1}, \ldots, \mathcal{X}_{s}$ where $\operatorname{lp}(f)=\mathcal{X}_{i} \operatorname{lp}\left(f_{i}\right)$ for all $i$ such that $c_{i} \neq 0$ and $\operatorname{lt}(f)=c_{1} \mathcal{X}_{1} \operatorname{lt}\left(f_{1}\right)+\ldots+c_{s} \mathcal{X}_{s} \operatorname{lt}\left(f_{s}\right)$.

(b) We say that $f$ can be reduced to $h$ modulo $F$, denoted by $f \stackrel{F}{\longrightarrow} h$, if and only if there exist polynomials $h_{1}, \ldots, h_{\ell-1} \in \mathbb{Z}_{n}\left[X_{1}, \ldots, X_{t}\right]$ such that $f \stackrel{F}{\longrightarrow} h_{1} \stackrel{F}{\longrightarrow} h_{2} \stackrel{F}{\longrightarrow} \ldots \stackrel{F}{\longrightarrow} h_{\ell-1} \stackrel{F}{\longrightarrow} h$.

(c) A polynomial $h$ is called minimal with respect to $F$ if $h$ cannot be reduced modulo $F$.

(d) We call h a (minimal) residue of $f$ modulo $F$, denoted by $h=f \bmod F$, if $f \stackrel{F}{\longrightarrow} h$ and $h$ is minimal.

Note that there is an efficient algorithm computing a minimal residue of a polynomial $f$ modulo a set $F$ (provided that the representation of $f$ and $F$ is efficient) according to the above definition. For instance, see Algorithm 4.1.1 in [AL94].

Definition 5 (Gröbner Basis). Let $J$ be an ideal in $\mathbb{Z}_{n}\left[X_{1}, \ldots, X_{t}\right]$ and $G=\left\{g_{1}, \ldots, g_{s}\right\}$ be a set of non-zero polynomials such that $\langle G\rangle=J$. Then $G$ is called a Gröbner basis for $J$ if for any polynomial $f \in \mathbb{Z}_{n}\left[X_{1}, \ldots, X_{t}\right]$ we have

$$
f \in J \Longleftrightarrow f \bmod G=0 .
$$

Fortunately, there always exists an ideal basis with this special property, as stated by Lemma 5 . However, note that given an arbitrary ideal basis, a Gröbner basis for the corresponding ideal is not always easy to compute, see Section 7. In the following we always assume that Gröbner bases for the considered ideals are given.

Lemma 5. Let $J$ be a non-zero ideal of $\mathbb{Z}_{n}\left[X_{1}, \ldots, X_{t}\right]$, then $J$ has a finite Gröbner basis. 
The following lemma is crucial for proving that (similar to the $\mathbb{Z}_{n}$-case) an element $f \in R \cong$ $R_{1} \times \ldots \times R_{k}$ that is congruent to zero over a component $R_{i}$ but not congruent to zero over another component $R_{j}$ (cf. Lemma 4) helps in factoring $n$. Observe that Lemma 6 requires that the leading coefficients of all given Gröbner basis elements are units. For our purposes, this is not a restriction at all but a reasonable assumption since otherwise the given representation of $R$ would immediately reveal a factor of $n$. A proof for this lemma based on the notion of syzygies can be found in Appendix A.

Lemma 6. Let $A=\mathbb{Z}_{n}\left[X_{1}, \ldots, X_{t}\right]$ and $n=\prod_{i=1}^{k} p_{i}^{e_{i}}$. Furthermore, let $G=\left\{g_{1}, \ldots, g_{s}\right\}$ be a Gröbner basis for the ideal $J=\left\langle g_{1}, \ldots, g_{s}\right\rangle$ in $A$ such that $\operatorname{lc}\left(g_{i}\right) \in \mathbb{Z}_{n}^{*}$ for all $1 \leq i \leq s$. Then for each $1 \leq \ell \leq s$ the set $G_{\ell}=\left\{p_{\ell}^{e_{\ell}}, g_{1}, \ldots, g_{s}\right\}$ is a Gröbner basis for the ideal $J_{\ell}=\left\langle p_{\ell}^{e_{\ell}}, g_{1}, \ldots, g_{s}\right\rangle$ in $A$.

\subsection{The Relation between BBRE and IF for $\mathbb{Z}_{n}\left[X_{1}, \ldots, X_{t}\right] / J$}

We are going to lift our reduction from the special case $R=\mathbb{Z}_{n}$ to the more general case of finite multivariate polynomials rings $R=\mathbb{Z}_{n}\left[X_{1}, \ldots, X_{t}\right] / J$, where $J$ is given by a Gröbner basis. Let $n=\prod_{i=1}^{k} p_{i}^{e_{i}}$. In the case $R=\mathbb{Z}_{n}$ our factoring algorithm was successful if it was able to find an element $a \in R$ such that $a \in\left\langle p_{i}^{e_{i}}\right\rangle$ and $a \notin\left\langle p_{j}^{e_{j}}\right\rangle$ for some $1 \leq i<j \leq k$. The following theorem shows that a generalization of this fact holds for residue class rings modulo polynomial ideals given by Gröbner basis.

Theorem 2. Let $A=\mathbb{Z}_{n}\left[X_{1}, \ldots, X_{t}\right]$ where $n=\prod_{i=1}^{k} p_{i}^{e_{i}}$ and $k \geq 2$. Furthermore, let $G=$ $\left\{g_{1}, \ldots, g_{s}\right\}$ be a Gröbner basis for the ideal $J=\left\langle g_{1}, \ldots, g_{s}\right\rangle$ in A such that $\operatorname{lc}\left(g_{i}\right) \in \mathbb{Z}_{n}^{*}$ for all $1 \leq i \leq s$. Assume an element $f \in A$ is given, such that $f \in J_{i}=\left\langle p_{i}^{e_{i}}, g_{1}, \ldots, g_{s}\right\rangle$ and $f \notin J_{j}=$ $\left\langle p_{j}^{e_{j}}, g_{1}, \ldots, g_{s}\right\rangle$ for some $1 \leq i<j \leq k$. Then computing $\operatorname{gcd}(\operatorname{lc}(r), n)$, where $r=f \bmod G$, yields a non-trivial factor of $n$.

Proof. First of all, observe that since $f \notin J_{j}$ we have that $f \notin J$ and so $r=f \bmod G$ is not zero by Definition 5 . Since $r$ is a minimal residue and the leading coefficients $\operatorname{lc}\left(g_{i}\right)$ of all Gröbner basis elements are units, it follows by Definition 4 that the leading monomial $\operatorname{lp}(r)$ of $r$ is not divisible by any leading monomial $\operatorname{lp}\left(g_{i}\right)$ of a Gröbner basis element. Otherwise, $r$ would be reducible to $r \stackrel{G}{\longrightarrow} r-\operatorname{lc}\left(g_{i}\right)^{-1} \operatorname{lc}(r) \mathcal{X}_{i} g_{i}$, where $\mathcal{X}_{i} \operatorname{lp}\left(g_{i}\right)=\operatorname{lp}(r)$, using some $g_{i}$ such that $\operatorname{lp}\left(g_{i}\right)$ divides $\operatorname{lp}(r)$. Moreover, by Lemma 6 , the set $G_{i}=\left\{p_{i}^{e_{i}}, g_{1}, \ldots, g_{s}\right\}$ is a Gröbner basis for the ideal $J_{i}$. Thus, since $f \in J_{i}$ also $r \in J_{i}$ and the reduction of $r$ modulo $G_{i}$ would yield the minimal residue zero. As the leading monomial $\operatorname{lp}(r)$ is not divisible by any $\operatorname{lp}\left(g_{i}\right)$, the leading coefficient $\operatorname{lc}(r)$ must be divisible by $p_{i}^{e_{i}}$. Since $r \neq 0$ and $\operatorname{lc}(r) \not \equiv 0 \bmod n$ (by definition of the leading coefficient) computing $\operatorname{gcd}(\operatorname{lc}(r), n)$ yields a non-trivial factor of $n$.

The following example illustrates the result captured by the above theorem. Moreover, it shows that in the case where $G$ is not a Gröbner basis, elements $f$ satisfying the properties from Theorem 2 seem not to reveal a factor of $n$ by considering their residues modulo $G$.

Example 1. Consider the finite ring $R=\mathbb{Z}_{225}\left[X_{1}, X_{2}\right] / J$ where $225=3^{2} \cdot 5^{2}$ and $J=\left\langle X_{1} X_{2}+\right.$ $\left.1, X_{2}^{2}+224\right\rangle$. Let us use the lexicographic order where $X_{1}>X_{2}$ for polynomial reduction. Note that $F=\left\{X_{1} X_{2}+1, X_{2}^{2}+224\right\}$ is a generating set, but not a Gröbner basis for $J$ with respect to this order. Furthermore, not that $G=\left\{X_{1}+X_{2}, X_{2}^{2}+224\right\}$ is a Gröbner basis for $J$. Let $f=X_{1}^{4} X_{2}^{2}+224 X_{1}^{4}+2 X_{1}+7 X_{2}^{2}+38 X_{2}+218=\left(4 X_{2}\right)\left(3^{2}\right)+\left(X_{1}^{4}+7\right)\left(X_{2}^{2}+224\right)+2\left(X_{1}+X_{2}\right) \bmod 225$. It holds that $f \in J_{1}=\left\langle 3^{2}, X_{1}+X_{2}, X_{2}^{2}+224\right\rangle$ but $f \notin J_{2}=\left\langle 5^{2}, X_{1}+X_{2}, X_{2}^{2}+224\right\rangle$. The polynomial 
$f$ can be reduced modulo $F$ to the minimal residue $f \bmod F=2 X_{1}+7 X_{2}^{2}+38 X_{2}+218=$ $f-\left(X_{1}^{4}+7\right)\left(X_{2}^{2}+224\right) \bmod 225$. It is easy to see that no coefficient of this residue share a nontrivial factor with 225. Modulo the Gröbner basis $G$ the polynomial $f$ can be reduced to the minimal residue $r=f \bmod G=36 X_{2}=f-\left(X_{1}^{4}+7\right)\left(X_{2}^{2}+224\right)-2\left(X_{1}+X_{2}\right) \bmod 225$. Computing $\operatorname{gcd}(\operatorname{lc}(r), 225)=9$ yields a non-trivial factor of 225 .

The above fact allows us to formulate and prove a theorem similar to Theorem 1 .

Theorem 3. Let $R:=\mathbb{Z}_{n}\left[X_{1}, \ldots, X_{t}\right] / J$ for some integer $n$ having at least two different prime factors and ideal $J$ in $\mathbb{Z}_{n}\left[X_{1}, \ldots, X_{t}\right]$. Assume a Gröbner basis $G=\left\{g_{1}, \ldots, g_{s}\right\}$ for $J$ is given. Let $\mathcal{A}$ be an algorithm for the BBRE problem that performs at most $m \leq|R|$ operations on $R^{\sigma}$. Assume that $\mathcal{A}$ solves the BBRE problem with probability $\epsilon$. Then there is an algorithm $\mathcal{B}$ having white-box access to $\mathcal{A}$ that finds a factor of $n$ with probability at least

$$
\frac{\epsilon-\frac{1}{n}}{(m+t+2)(m+t+1)}
$$

by running $\mathcal{A}$ once and performing an additional amount of $O\left((m+t)^{2}\right)$ random choices and $O\left(m(m+t)^{2}\right)$ operations on $R$ as well as $O\left((m+t)^{2}+s\right)$ gcd computations on $\log _{2}(n)$-bit integers. $^{2}$

Proof. We adapt the proof of Theorem 1. The description of the original and the simulation game almost carries over completely by setting $R:=\mathbb{Z}_{n}\left[X_{1}, \ldots, X_{t}\right] / J$. There are only a few slight technical differences concerning the oracles $\mathcal{O}$ and $\mathcal{O}_{\text {sim }}$ considered in the original game (cf. Definition 3 ) and the simulation game:

- The list $L$ maintained by both oracles is initialized with the $t+1$ generating elements $1, X_{1}, \ldots, X_{t}$ of $R$, and with the variable $X$. As before, computed ring elements are represented by polynomials in $R[X]=\left(\mathbb{Z}_{n}\left[X_{1}, \ldots, X_{t}\right] / J\right)[X]$.

- Whenever an element $P \in R[X]$ is appended to the list $L$, say as element $L_{j}=P, \mathcal{O}^{\prime}$ checks whether there exists an element $L_{i} \in L$ such that $\left(L_{i}-L_{j}\right)(x) \in J$ which is equivalent to checking whether the residue $r=\left(L_{i}-L_{j}\right)(x) \bmod G$ is the zero polynomial over $\mathbb{Z}_{n}$. Instead of using the given secret $x$ in the above evaluation, the simulation oracle $\mathcal{O}_{\text {sim }}$ performs this check using a new random element $x_{i, j} \in R$ for each difference polynomial $L_{i}-L_{j}(i<j \in\{1, \ldots,|L|\})$.

The rest of the description of the games applies unchanged. Let the events $\mathbf{S}, \mathbf{S}_{\text {sim }}$ and $\mathbf{F}$ be defined analogously to the case $R=\mathbb{Z}_{n}$. We are left with deriving bounds for the success probability $\operatorname{Pr}\left[\mathbf{S}_{\text {sim }}\right]$ in the simulation game and for the probability $\operatorname{Pr}[\mathbf{F}]$ of a simulation failure. This also works similarly to the previous case except for some technical differences.

Bounding the Probability of Success in the Simulation Game. All computations in the simulation game are independent of the uniformly random element $x$. Thus, the algorithm $\mathcal{A}$ can only guess $x$, resulting in

$$
\operatorname{Pr}\left[\mathbf{S}_{\text {sim }}\right] \leq \frac{1}{|R|} \leq \frac{1}{n}
$$

\footnotetext{
${ }^{2}$ We count the addition/multiplication of two ring elements together with the reduction modulo $G$ as one ring operation.
} 
Bounding the Probability of a Simulation Failure. Again let $\mathfrak{D}:=\left\{L_{i}-L_{j}|1 \leq i<j \leq| L \mid\right\}$ denote the set of all non-trivial differences of polynomials in $L$ after a run of $\mathcal{A}$, and let $\Delta$ be some (fixed) element of $\mathfrak{D}$. Let $n=\prod_{i=1}^{k} p_{i}^{e_{i}}$ be the prime factor decomposition of $n$, then $R$ has a prime power decomposition into

$$
R \cong \mathbb{Z}\left[X_{1}, \ldots, X_{t}\right] /\left\langle p_{1}^{e_{1}}, G\right\rangle \times \ldots \times \mathbb{Z}\left[X_{1}, \ldots, X_{t}\right] /\left\langle p_{k}^{e_{k}}, G\right\rangle
$$

according to Lemma 4 . Let

$$
\nu_{i}:=\frac{\left|\left\{a \in R \mid \Delta(a) \in\left\langle p_{i}^{e_{i}}, G\right\rangle\right\}\right|}{|R|}
$$

be the probability that $\Delta(a) \in\left\langle p_{i}^{e_{i}}, G\right\rangle$ for a uniformly random element $a \in R$. Using this redefinition of $\nu_{i}$, the probability $\operatorname{Pr}\left[\mathbf{F}_{\Delta}\right]$ that $\Delta$ causes a simulation failure is given by Equation 4.

By Theorem 2, $\Delta$ reveals a factor of $n$ if we can find an element $a \in R$ such that $\Delta(a) \in\left\langle p_{i}^{e_{i}}, G\right\rangle$ and $\Delta(a) \notin\left\langle p_{j}^{e_{j}}, G\right\rangle$ for some $1 \leq i<j \leq k$. In this case computing $\operatorname{gcd}(\operatorname{lc}(\Delta(a) \bmod G), n)$ yields a non-trivial factor provided that $\operatorname{lc}(g) \in \mathbb{Z}_{n}^{*}$ for all $g \in G$. The probability $\operatorname{Pr}\left[\mathbf{D}_{\Delta}\right]$ of finding such an element $a$ at random is given in terms of $\nu_{i}$ by Equation 5. Clearly, we again obtain the following relation between $\operatorname{Pr}\left[\mathbf{D}_{\Delta}\right]$ and $\operatorname{Pr}\left[\mathbf{F}_{\Delta}\right]$ in this case:

$$
2 \operatorname{Pr}\left[\mathbf{D}_{\Delta}\right] \geq \operatorname{Pr}\left[\mathbf{F}_{\Delta}\right]
$$

The Factoring Algorithm. Consider an algorithm $\mathcal{B}$ that first tries to find a factor of $n$ by computing $\operatorname{gcd}(\operatorname{lc}(g), n)$ for all $g \in G$. Then it runs the BBRE algorithm $\mathcal{A}$ on an arbitrary instance of the BBRE problem over $R$ and records the sequence of queries that $\mathcal{A}$ issues. For each $\Delta \in \mathfrak{D}$ the algorithm $\mathcal{B}$ chooses a new random element $a \in R$, and computes $\operatorname{gcd}(\operatorname{lc}(\Delta(a) \bmod G), n)$. There are at most $(m+t+2)(m+t+1) / 2$ such polynomials and each can be evaluated using at most $m+1$ ring operations. Thus, in total $\mathcal{B}$ chooses $O\left((m+t)^{2}\right)$ random elements and performs $O\left(m(m+t)^{2}\right)$ operations on $R$ as well as $O\left((m+t)^{2}+s\right)$ gcd computations on $\log _{2}(n)$-bit integers.

Let $\mathbf{D}$ denote the event that $\mathcal{B}$ finds a factor of $n$. The total probability of simulation failure $\operatorname{Pr}[\mathbf{F}]$ is upper bounded by

$$
\operatorname{Pr}[\mathbf{F}] \leq \sum_{\Delta \in \mathfrak{D}} \operatorname{Pr}\left[\mathbf{F}_{\Delta}\right] \leq 2 \sum_{\Delta \in \mathfrak{D}} \operatorname{Pr}\left[\mathbf{D}_{\Delta}\right] \leq(m+t+2)(m+t+1) \operatorname{Pr}[\mathbf{D}]
$$

Therefore, the probability of finding a factor of $n$ with this algorithm is at least

$$
\operatorname{Pr}[\mathbf{D}] \geq \frac{\epsilon-\frac{1}{n}}{(m+t+2)(m+t+1)} .
$$

Note that univariate polynomial quotient rings of the form $\mathbb{Z}_{n}\left[X_{1}\right] / J$ for some ideal $J$ in $\mathbb{Z}_{n}\left[X_{1}\right]$ are covered by Theorem 3 as a special case. A Gröbner basis for $J$ can always be easily determined: If $J$ is given by a single polynomial $g$ we are done. In the case where $J$ is described by a set of polynomials $\left\{g_{1}, \ldots, g_{s}\right\}$, a unique polynomial $g$ generating $J$ can be computed as $g=\operatorname{gcd}\left(g_{1}, \ldots, g_{s}\right)$. Furthermore, we can use the standard polynomial division algorithm (for univariate polynomials) to implement reduction modulo $g$.

Let $(n, t, G) \leftarrow \operatorname{RGen}(\kappa)$ be a ring instance generator that on input of a security parameter $\kappa$ (in unary representation) outputs the description $(n, t, G)$ of a $\operatorname{ring} R=\mathbb{Z}_{n}\left[X_{1}, \ldots, X_{t}\right] /\langle G\rangle$, where $n$ is an integer consisting of at least two different primes, $t$ specifies the number of indeterminates, and $G$ is a Gröbner basis for the ideal $\langle G\rangle$. Note that the parameters $n, t$, as well as the Gröbner basis 
(i.e., $|G|$ and the individual elements of the Göbner basis) may all depend on $\kappa$. Let us assume that addition, subtraction, multiplication, reduction modulo $G$ as well as sampling random elements in the rings $R$ takes polynomial-time in $\kappa$. Furthermore, let there exist a non-constant polynomial $q(\cdot)$ over $\mathbb{N}$ such that for all $\kappa$ and possible outputs $(n, t, G) \leftarrow \operatorname{RGen}(\kappa)$ it holds that $\log _{2}(n) \geq q(\kappa)$. Then Theorem 3 provides a polynomial-time (in $\kappa$ ) reduction from finding a factor of $n$ to the black-box ring extraction problem for the family of rings described by RGen.

\section{Extending our Reduction to Rings in Basis Representation}

In this section, we show that our reductions also works for rings that are given in basis representation. A basis representation of a ring $R$ is a tuple

$$
\left(n_{1}, \ldots, n_{t},\left(c_{i, j, k}\right)_{1 \leq i, j, k \leq t}\right)
$$

where $n_{1}, \ldots, n_{t}$ are the additive orders of elements $b_{1}, \ldots, b_{t} \in R$ generating $(R,+)$ and the $\left.c_{i, j, k}\right) \in \mathbb{Z}_{n_{k}}$ are integers describing the effect of multiplication on the $b_{i}$ via

$$
b_{i} b_{j}:=\sum_{k=1}^{t} c_{i, j, k} b_{k} .
$$

Thus, elements of $R$ are represented by tuples

$$
\left(r_{1}, \ldots, r_{t}\right) \in \mathbb{Z}_{n_{1}} \times \ldots \times \mathbb{Z}_{n_{t}} .
$$

The addition of two elements $r=\left(r_{1}, \ldots, r_{t}\right)$ and $s=\left(s_{1}, \ldots, s_{t}\right)$ in this representation is defined by their componentwise addition, i.e.,

$$
r+s=\left(r_{1}+s_{1}, \ldots, r_{t}+s_{t}\right) .
$$

Multiplication is defined by

$$
r \cdot s=\sum_{1 \leq i, j \leq t}\left(r_{i} s_{j} c_{i, j, 1}, \ldots, r_{i} s_{j} c_{i, j, t}\right) .
$$

The two elements are equal, which is denoted by $r \equiv s$, if and only if $r_{i} \equiv s_{i} \bmod n_{i}$ for all $1 \leq i \leq t$. The elements $b_{1}=(1,0, \ldots, 0), b_{2}=(0,1,0, \ldots, 0), \ldots, b_{t}=(0, \ldots, 0,1)$ represent an additive basis of $R$.

Remark 3. Note that the ring $R$ is not necessarily ring-isomorphic to the product ring $\mathbb{Z}_{n_{1}} \times \ldots \times \mathbb{Z}_{n_{t}}$ since multiplication is defined differently. However, if the basis representation satisfies

$$
c_{i, j, k}= \begin{cases}1, & i=j=k \\ 0, & \text { else }\end{cases}
$$

then such an isomorphism exists. Indeed, the basis representation of $R$ essentially corresponds to the canonical representation $\mathbb{Z}_{n_{1}} \times \ldots \times \mathbb{Z}_{n_{t}}$. As a special case, we obtain $R=\mathbb{Z}_{n}$ by setting $n_{1}=n$ and $t=1$.

The size of this representation is bounded by $O\left((\log |R|)^{3}\right)$. Thus, choosing this representation for general finite commutative rings might be of interest for cryptographic purposes. However, the integers $n_{i}$ - which need to be known - are factors of the characteristic $n$. Hence, in the following we only need to consider the case where all $n_{i}$ are equal to the characteristic $n$, since otherwise at least one of the $n_{i}$ is a non-trivial factor of $n$ and thus the representation would not hide the factorization of $n$. Theorem 4 formulates our reduction for this case. 
Theorem 4. Let $R=\left(n_{1}, \ldots, n_{t},\left(c_{i, j, k}\right)_{1 \leq i, j, k \leq t}\right)$ be a finite commutative unitary ring of characteristic $n$ in basis representation such that $n_{1}=\cdots=n_{t}=n$. Let $\mathcal{A}$ be an algorithm for the BBRE problem that performs at most $m \leq|R|$ operations on $R^{\sigma}$ and solves the BBRE problem with probability $\epsilon$. Then there is an algorithm $\mathcal{B}$ having white-box access to $\mathcal{A}$ that finds a factor of $n$ with probability at least

$$
\frac{\epsilon-\frac{1}{n}}{(m+t+2)(m+t+1)}
$$

by running $\mathcal{A}$ once and performing an additional amount of $O\left((m+t)^{2}\right)$ random choices and $O\left(m(m+t)^{2}\right)$ operations on $R$ as well as $O\left((m+t)^{2}\right)$ gcd computations on $\log _{2}(n)$-bit integers.

Proof. Let $n=\prod_{i=1}^{k} p_{i}^{e_{i}}$ be the prime factor decomposition of $n$. Then according to Corollary 1 $R$ can be decomposed into a direct product $R_{1} \times \ldots \times R_{k}$ of rings such that $R_{i}$ has characteristic $p_{i}^{e_{i}}$ for $1 \leq i \leq k$. Let $\phi_{i}: R \rightarrow R_{i}$ be the surjective homomorphisms projecting $R$ to the $i$-th component $R_{i}$.

Then we can again setup a simulation game that is similar to the one in the proofs of Theorems 1 and 3. In particular, we get the well-known relation

$$
\begin{aligned}
\operatorname{Pr}\left[\mathbf{F}_{\Delta}\right] & =2 \operatorname{Pr}_{a \in_{U} R}[\Delta(a)=0 \in R]\left(1-\operatorname{Pr}_{a \in_{U} R}[\Delta(a)=0 \in R]\right) \\
& \leq 2 \operatorname{Pr}_{a \in_{U} R}\left[\exists i, j: \phi_{i}(\Delta(a))=0 \in R_{i} \text { and } \phi_{j}(\Delta(a)) \neq 0 \in R_{j}\right] \\
& =2 \operatorname{Pr}\left[\mathbf{D}_{\Delta}\right]
\end{aligned}
$$

It remains to show that the basis representation of an element $\Delta(a)$ projecting to zero in a component $R_{i}$ and not to zero in a component $R_{j}$ indeed reveals a factor of $n$. Let

$$
r=\left(r_{1}, \ldots, r_{t}\right),
$$

where $r_{i} \in \mathbb{Z}_{n}$, denote the basis representation of $\Delta(a)$. Then we have $r \not \equiv(0, \ldots, 0)$. Furthermore, let $I:=\left\{i \mid \phi_{i}(\Delta(a)) \neq 0 \in R_{i}\right\}$ and $\rho:=\prod_{i \in I} p_{i}^{e_{i}}$. The integer $\rho$ is a multiple of the characteristic of $R_{i}$ and thus we obtain $\phi_{i}(\rho \Delta(a))=0 \in R_{i}$ for each $i \in I$. Hence, $\rho \Delta(a)$ is equal to the zero element in the ring $R$ which would lead to the basis representation

$$
\rho r=\left(\rho r_{1}, \ldots, \rho r_{t}\right) \equiv(0, \ldots, 0)
$$

From that we can observe that each non-zero component $r_{i}$ must already be a zero divisor in $\mathbb{Z}_{n}$ and so computing $\operatorname{gcd}\left(r_{i}, n\right)$ yields a factor.

As before, our factoring algorithm $\mathcal{B}$ chooses a new random element $a \in R$ for each $\Delta \in \mathfrak{D}$ and computes $\operatorname{gcd}\left(r_{i}, n\right)$, where $r_{i}$ is a non-zero component in the basis representation of $\Delta(a)$.

\section{Extending our Reduction to Product Rings}

Our reduction naturally extends to product rings where at least one component ring is given in a representation already covered by Theorem 3, or 4 . Note that in the following theorem the integer $n$ is not necessarily the characteristic of the product ring $R$.

Theorem 5. Let $R:=R_{1} \times \ldots \times R_{\ell}$ be the direct product of finitely many rings where $R_{1}=$ $\mathbb{Z}_{n}\left[X_{1}, \ldots, X_{t}\right] / J$ or $R_{1}=\left(n_{1}, \ldots, n_{t},\left(c_{i, j, k}\right)_{1 \leq i, j, k \leq t}\right)$. Let the integer $n$ consist of at least two different prime factors, let $n_{1}=\cdots=n_{t}=n$, and let the ideal $J$ be given by a Gröbner basis 
$G=\left\{g_{1}, \ldots, g_{s}\right\}$. Let $\mathcal{A}$ be an algorithm for the BBRE problem that performs at most $m \leq|R|$ operations on $R^{\sigma}$. Assume that $\mathcal{A}$ solves the BBRE problem with probability $\epsilon$. Then there is an algorithm $\mathcal{B}$ having white-box access to $\mathcal{A}$ that finds a factor of $n$ with probability at least

$$
\frac{\epsilon-\frac{1}{n}}{(m+t+2)(m+t+1)}
$$

by running $\mathcal{A}$ once and performing an additional amount of $O\left((m+t)^{2}\right)$ random choices and $O\left(m(m+t)^{2}\right)$ operations on $R_{1}$ as well as $O\left((m+t)^{2}+s\right) \operatorname{gcd}$ computations on $\log _{2}(n)$-bit integers (where $s=0$ if $R_{1}$ is not given in polynomial representation).

Proof. Given $\sigma(x) \in R^{\sigma}$ where $x=\left(x_{1}, \ldots, x_{\ell}\right)$ is uniformly chosen from $R=R_{1} \times \ldots \times R_{\ell}$ the algorithm $\mathcal{A}$ finds $x$, i.e., all components $x_{1}, \ldots, x_{\ell}$, with probability $\epsilon$. Thus, given $\sigma(x)$ it outputs an element $\left(y_{1}, \ldots, y_{\ell}\right)$ such that $y_{1}=x_{1}$ with probability at least $\epsilon$. Furthermore, observe that choosing an element $x$ uniformly at random from $R$ is equivalent to choosing each component $x_{i}$ uniformly at random from $R_{i}$. Thus, informally speaking, $\mathcal{A}$ solves the BBRE problem over each component ring separately. Hence, we can simply apply the ideas from the proofs of Theorems 1, 3, and 4 to this case, namely to the component $R_{1}$.

More precisely, we can just generalize the description of the original and the simulation game to product rings (which is a straightforward task) except for one modification: instead of making the computations in the simulation game independent of $x$, i.e., all components $x_{1}, \ldots, x_{\ell}$, we make them only independent of the component $x_{1}$. That means, when determining encodings, the simulation oracle $\mathcal{O}_{\text {sim }}$ still evaluates polynomials over $R_{2}, \ldots, R_{\ell}$ with the given inputs $x_{2}, \ldots, x_{\ell}$ exactly as $\mathcal{O}^{\prime}$ does and only chooses new random elements for evaluating polynomials over $R_{1}$. In this way, only the modification over $R_{1}$ can lead to a difference in the behavior of $\mathcal{O}_{\text {sim }}$ and $\mathcal{O}^{\prime}$ and so we can define the event of a simulation failure as before.

The success probability of $\mathcal{A}$ in the simulation game is upper bounded by the probability that it outputs $x_{1}$ which is at most $\frac{1}{n}$. The probability of a simulation failure can be bounded exactly as before.

Similarly, the factoring algorithm $\mathcal{B}$ runs $\mathcal{A}$ on some instance of the BBRE problem over $R$ and records the sequence of queries. The corresponding difference polynomials can be seen as polynomials over $R_{1}$ and so $\mathcal{B}$ does the same steps as the factoring algorithms described in the previous proofs depending on the representation of $R_{1}$.

Hence, we conclude that we obtain the same relation between the success probability of $\mathcal{A}$ and $\mathcal{B}$ and the same number of additional operations $\mathcal{B}$ performs as in the simple cases.

\section{Implications for General Rings}

In this section we like to clarify the implications of our result to general finite commutative rings with unity. Our reduction seems to apply to any representation of such a ring $R$ that does not immediately reveal a factor of the characteristic of $R$ and allows for efficient computations, as explained in the following.

There are essentially three ways to represent a finite commutative ring [AS05], namely the table representation, the basis representation, and the polynomial representation. Using the table representation for a ring $R$ means to represent $R$ by an addition and multiplication table which requires $O\left(|R|^{2}\right)$ space. Hence, considering ring sizes of cryptographic interest this type of representation can be immediately excluded from the list of possible representations.

We considered the basis representation of a ring in Section 5 and provided a reduction for all cases where the representation itself does not immediately leak a factor of the ring characteristic. 
A reduction for rings in polynomial representation was given in Section 4. Unfortunately, to make our reduction work for this representation the ideal $J$ must not be given by an arbitrary basis but a Gröbner basis. If we are given a basis other than a Gröbner, we theoretically could compute a Gröbner basis from this input using a variant of Buchberger's algorithm for Noetherian rings [AL94] (or corresponding variants of Faugere's F4 [Fau99] and F5 [Fau02] algorithm). However, for Gröbner basis algorithms one still does not know an upper bound on their running times (even in the case when the coefficient space is a field). The work due to Mayr and Meier [MM82] implies that there are instances where constructing such a basis takes time in the order of $2^{2^{O(t)}}$, where $t$ is the number variables. Thus, we cannot give the factoring algorithm described in our reduction an arbitrary basis of the ideal $J$ as input and let it first compute a Gröbner basis, since there are cases where this computation easily exceeds the time needed to factor $n$ directly using the GNFS. So the reduction would be meaningless. Hence, our result only holds for families of rings in polynomial representation where a Gröbner basis for $J$ is given or known to be efficiently computable.

This is the "bad" news. The "good" news is that in order to be able to perform equality checks between ring elements in this representation efficiently - which corresponds to solving instances of the ideal membership problem - there is currently no other way than providing a Gröbner basis for the ideal $J$. However, we note that apparently there are rings where this representation does not allow for efficient computations (e.g., because $J$ cannot be efficiently represented by a Gröbner basis).

Acknowledgments. We like to thank Roberto Avanzi, Lothar Gerritzen, and Gregor Leander for helpful discussions as well as Daniel Brown for pointing out an error in a previous version of the paper.

\section{References}

[AL94] William Adams and Philippe Loustaunau. An introduction to Gröbner bases. Graduate Studies in Math. Vol. 3. Oxford University Press, 1994.

[AS05] Manindra Agrawal and Nitin Saxena. Automorphisms of finite rings and applications to complexity of problems. In Volker Diekert and Bruno Durand, editors, 22nd Annual Symposium on Theoretical Aspects of Computer Science, STACS 2005, volume 3404 of Lecture Notes in Computer Science, pages 1-17. Springer, 2005.

[Bac84] Eric Bach. Discrete logarithms and factoring. Technical Report UCB/CSD-84-186, EECS Department, University of California, Berkeley, Jun 1984.

[BL96] Dan Boneh and Richard J. Lipton. Algorithms for black-box fields and their application to cryptography (extended abstract). In Neal Koblitz, editor, Advances in Cryptology - CRYPTO '96, 16th Annual International Cryptology Conference, volume 1109 of Lecture Notes in Computer Science, pages 283-297. Springer, 1996.

[Buc65] Bruno Buchberger. Ein Algorithmus zum Auffinden der Basiselemente des Restklassenringes nach einem nulldimensionalen Polynomideal (An Algorithm for Finding the Basis Elements in the Residue Class Ring Modulo a Zero Dimensional Polynomial Ideal). PhD thesis, Mathematical Institute, University of Innsbruck, Austria, 1965. (English translation in Journal of Symbolic Computation, 2004).

[dB88] Bert den Boer. Diffie-Hellman is as strong as discrete log for certain primes. In Shafi Goldwasser, editor, Advances in Cryptology - CRYPTO '88, 8th Annual International Cryptology Conference, volume 403 of Lecture Notes in Computer Science, pages 530-539. Springer, 1988.

[ElG85] Taher ElGamal. A public key cryptosystem and a signature scheme based on discrete logarithms. IEEE Transactions on Information Theory, 31(4):469-472, 1985.

[Fau99] Jean Charles Faugère. A new efficient algorithm for computing Gröbner bases $\left(F_{4}\right)$. Journal of Pure and Applied Algebra, 139(1-3):61-88, 1999.

[Fau02] Jean Charles Faugère. A new efficient algorithm for computing Gröbner bases without reduction to zero $\left(F_{5}\right)$. In International Symposium on Symbolic and Algebraic Computation - ISSAC 2002, pages 75-83. ACM, 2002.

[Gri99] Pierre A. Grillet. Algebra (Pure and Applied Mathematics). John Wiley \& Sons Inc., 1999. 
[Lan02] Serge Lang. Algebra. Springer-Verlag, 2002.

[LR06] Gregor Leander and Andy Rupp. On the equivalence of RSA and factoring regarding generic ring algorithms. In Xuejia Lai and Kefei Chen, editors, Advances in Cryptology - ASIACRYPT 2007, 13th International Conference on the Theory and Application of Cryptology and Information Security, volume 4284 of Lecture Notes in Computer Science, pages 241-251. Springer, 2006.

[Mau94] Ueli Maurer. Towards the equivalence of breaking the Diffie-Hellman protocol and computing discrete algorithms. In Yvo Desmedt, editor, Advances in Cryptology - CRYPTO '94, 14th Annual International Cryptology Conference, volume 839 of Lecture Notes in Computer Science, pages 271-281. Springer, 1994.

[Mau05] Ueli Maurer. Abstract models of computation in cryptography. In Nigel P. Smart, editor, IMA Int. Conf., volume 3796 of Lecture Notes in Computer Science, pages 1-12. Springer, 2005.

[McD74] Bernard R. McDonald. Finite Rings with Identity. Marcel Dekker, New York, 1974.

[MM82] Ernst W. Mayr and Albert Meyer. The complexity of the word problems for commutative semigroups and polynomial ideals. Advances in Mathematics, 46:305-329, 1982.

[MR07] Ueli Maurer and Dominik Raub. Black-box extension fields and the inexistence of field-homomorphic one-way permutations. In Kaoru Kurosawa, editor, Advances in Cryptology - ASIACRYPT 2007, 13th International Conference on the Theory and Application of Cryptology and Information Security, volume 4833 of Lecture Notes in Computer Science, pages 427-443. Springer, 2007.

[MW98] Ueli M. Maurer and Stefan Wolf. Lower bounds on generic algorithms in groups. In Kaisa Nyberg, editor, Advances in Cryptology - EUROCRYPT '98, International Conference on the Theory and Application of Cryptographic Techniques, volume 1403 of Lecture Notes in Computer Science, pages 72-84. Springer, 1998.

[Nec94] V. I. Nechaev. Complexity of a determinate algorithm for the discrete logarithm. Mathematical Notes, 55(2):165-172, 1994.

[Pai99] Pascal Paillier. Public-key cryptosystems based on composite degree residuosity classes. In Jacques Stern, editor, Advances in Cryptology - EUROCRYPT '99, International Conference on the Theory and Application of Cryptographic Techniques, volume 1592 of Lecture Notes in Computer Science, pages 223-238. Springer, 1999.

[Sho97] Victor Shoup. Lower bounds for discrete logarithms and related problems. In Walter Fumy, editor, Advances in Cryptology - EUROCRYPT '97, International Conference on the Theory and Application of Cryptographic Techniques, volume 1233 of Lecture Notes in Computer Science, pages 256-266. Springer, 1997.

[Sho04] Victor Shoup. Sequences of games: a tool for taming complexity in security proofs. Cryptology ePrint Archive, Report 2004/332, 2004. http://eprint.iacr.org/.

\section{A Detailed Proof of Lemma 6}

In this section we provide a proof for Lemma 6. Our proof is based on an alternative but equivalent definition of Gröbner bases using the notion of syzygies. We partly make use of definitions and theorems given in Chapters 3.2 and 4.2 of [AL94].

Throughout this section let $A=D\left[X_{1}, \ldots, X_{t}\right]$ where $D=\mathbb{Z}_{n}$ is a Noetherian ring. Let $I=$ $\left\langle f_{1}, \ldots, f_{s}\right\rangle$ be an ideal of $A$. Consider the $A$-module homomorphism

$$
\begin{aligned}
\phi: A^{s} & \rightarrow I \\
\left(h_{1}, \ldots, h_{s}\right) & \mapsto \sum_{i=1}^{s} h_{i} f_{i}
\end{aligned}
$$

Then it holds that $I \cong A^{s} / \operatorname{ker}(\phi)$. Based on $\phi$ a syzygy is defined as follows:

Definition 6 (Syzygy). The kernel of the map $\phi$ is called the syzygy module of the $1 \times s$ matrix $\left[f_{1} \ldots f_{s}\right]$ and is denoted by $\operatorname{Syz}\left(f_{1} \ldots f_{s}\right)$. An element $\left(h_{1}, \ldots, h_{s}\right) \in \operatorname{Syz}\left(f_{1} \ldots f_{s}\right)$ is called a syzygy of $\left[f_{1} \ldots f_{s}\right]$ and satisfies

$$
h_{1} f_{1}+\cdots+h_{s} f_{s}=0 .
$$

Definition 7 (Homogeneous Syzygy). Let power products $\mathcal{X}_{1}, \ldots, \mathcal{X}_{s}$ and non-zero elements $c_{1}, \ldots, c_{s} \in D$ be given. For a power product $\mathcal{X}$, we call a syzygy $h=\left(h_{1}, \ldots, h_{s}\right) \in \operatorname{Syz}\left(c_{1} \mathcal{X}_{1}, \ldots, c_{s} \mathcal{X}_{s}\right)$ homogeneous of degree $\mathcal{X}$ if $\operatorname{lt}\left(h_{i}\right)=h_{i}$, thus $h_{i}$ is a term itself, and $\mathcal{X}_{i} \operatorname{lp}\left(h_{i}\right)=\mathcal{X}$ for all $i$ such that $h_{i} \neq 0$. 
As $A$ is a Noetherian ring, $\operatorname{Syz}\left(c_{1} \mathcal{X}_{1}, \ldots, c_{s} \mathcal{X}_{s}\right)$ has a finite generating set of homogeneous syzygies. Moreover, by [AL94] we have the following equivalent characterization of Gröbner bases:

Theorem 6 (Theorem 4.2.3 [AL94]). Let $G=\left\{g_{1}, \ldots, g_{s}\right\}$ be a set of non-zero polynomials in A. Let $\mathfrak{B}$ be a homogeneous generating set for $\operatorname{Syz}\left(\operatorname{lt}\left(g_{1}\right) \ldots, \operatorname{lt}\left(g_{s}\right)\right)$. Then $G$ is a Gröbner basis for the ideal $\left\langle g_{1}, \ldots, g_{s}\right\rangle$ if and only if for all $\left(h_{1}, \ldots, h_{s}\right) \in \mathfrak{B}$ we have

$$
\sum_{i=1}^{s} h_{i} g_{i} \stackrel{G}{\longrightarrow}+0
$$

Our proof for Lemma 6 will essentially be based on the above theorem. However, before we can actually give this proof we need to introduce two of auxiliary lemmas.

Lemma 7. Let $\left\{f_{1}, \ldots, f_{s}\right\}$ be a basis for an ideal $I$ of $A$. For $1 \leq i \leq s$ let the leading term of $f_{i}$ be denoted by $\operatorname{lt}\left(f_{i}\right)=c_{i} \mathcal{X}_{i}$. If all leading coefficients $c_{i}$ are units in $D$ then

$$
\mathfrak{B}_{\mathrm{Syz}\left(\mathrm{lt}\left(f_{1}\right), \ldots, \operatorname{lt}\left(f_{s}\right)\right)}=\left\{S_{i j}=S_{j i}=\frac{\mathcal{X}_{i j}}{c_{i} \mathcal{X}_{i}} e_{i}-\frac{\mathcal{X}_{i j}}{c_{j} \mathcal{X}_{j}} e_{j} \mid 1 \leq i<j \leq s\right\},
$$

where $e_{1}, \ldots, e_{s}$ form the standard basis for $A^{s}$ and $\mathcal{X}_{i j}=\operatorname{lcm}\left(\mathcal{X}_{i}, \mathcal{X}_{j}\right)$, is a generating set for $\operatorname{Syz}\left(\operatorname{lt}\left(f_{1}\right), \ldots, \operatorname{lt}\left(f_{s}\right)\right)$ of homogeneous syzygies.

Proof. First of all, if $i \neq j$ then $\frac{\mathcal{X}_{i j}}{c_{i} \mathcal{X}_{i}} e_{i}-\frac{\mathcal{X}_{i j}}{c_{j} \mathcal{X}_{j}} e_{j}$ is a syzygy of $\operatorname{Syz}\left(\operatorname{lt}\left(f_{1}\right), \ldots, \operatorname{lt}\left(f_{s}\right)\right)$ as $\frac{\mathcal{X}_{i j}}{c_{i} \mathcal{X}_{i}} \operatorname{lt}\left(f_{i}\right)-$ $\frac{\mathcal{X}_{i j}}{c_{j} \mathcal{X}_{j}} \operatorname{lt}\left(f_{j}\right)=0$. Furthermore, the non-zero polynomials $\frac{\mathcal{X}_{i j}}{c_{i} \mathcal{X}_{i}}$ and $\frac{\mathcal{X}_{i j}}{c_{j} \mathcal{X}_{j}}$ are terms and the sygyzy $S_{i j}=S_{j i}$ is homogeneous of degree $\mathcal{X}_{i j}$ as $\mathcal{X}_{i} \cdot \frac{\mathcal{X}_{i j}}{c_{i} \mathcal{X}_{i}}=\mathcal{X}_{i j}=\mathcal{X}_{j} \cdot \frac{\mathcal{X}_{i j}}{c_{j} \mathcal{X}_{j}}$.

Therefore we need to prove that $\mathfrak{B}_{\operatorname{Syz}}\left(\operatorname{lt}\left(f_{1}\right), \ldots, \operatorname{lt}\left(f_{s}\right)\right)$ is a basis of the ideal $\operatorname{Syz}\left(\operatorname{lt}\left(f_{1}\right), \ldots, \operatorname{lt}\left(f_{s}\right)\right)$. Let $h=\left(h_{1}, \ldots, h_{s}\right) \in \operatorname{Syz}\left(\operatorname{lt}\left(f_{1}\right), \ldots, \operatorname{lt}\left(f_{s}\right)\right)$ then

$$
\sum_{i=1}^{s} h_{i} \operatorname{lt}\left(f_{i}\right)=\sum_{i=1}^{s} c_{i} \cdot \mathcal{X}_{i} \cdot h_{i}=\sum_{i=1}^{s} c_{i} \cdot \mathcal{X}_{i}\left(\sum_{k=1}^{d_{i}} a_{k}^{(i)} \mathcal{X}_{k}^{(i)}\right)=0
$$

for $h_{i}=\sum_{k=1}^{d_{i}} a_{k}^{(i)} \mathcal{X}_{k}^{(i)}$. Let $\mathcal{X}$ be any power product (in the variables $X_{1}, \ldots, X_{t}$ ). Then the coefficient of $\mathcal{X}$ in the polynomial $\sum_{i=1}^{s} c_{i} \cdot \mathcal{X}_{i} \cdot h_{i}$ must be zero. Let

$$
\left\{\mathcal{Y}_{1}, \ldots, \mathcal{Y}_{d}\right\}=\bigcup_{i=1}^{s}\left\{\mathcal{X}_{k}^{(i)} \mid 1 \leq k \leq d_{i}\right\}
$$

with $\mathcal{Y}_{1}<\cdots<\mathcal{Y}_{d}$. Then

$$
h_{i}=\sum_{k=1}^{d_{i}} a_{k}^{(i)} \mathcal{X}_{k}^{(i)}=\sum_{\ell=1}^{d} b_{\ell}^{(i)} \mathcal{Y}_{\ell} \text { with }\left\{\begin{array}{ll}
b_{\ell}^{(i)}=a_{k}^{(i)} & \text { if } \mathcal{Y}_{\ell}=\mathcal{X}_{k}^{(i)} \\
b_{\ell}^{(i)}=0 & \text { else }
\end{array} \text { for some index } 1 \leq k \leq d_{i} .\right.
$$


Moreover, let $\mathcal{X}$ be a power product and $1 \leq m_{i} \leq d$ be the index such that $\mathcal{X}_{i} \cdot \mathcal{Y}_{m_{i}}=\mathcal{X}$ for $1 \leq i \leq s$. Then $\sum_{i=1}^{s} c_{i} \cdot b_{m_{i}}=0$ and we have

$$
\begin{aligned}
\left(b_{m_{1}} \cdot \mathcal{Y}_{m_{1}}, \ldots, b_{m_{s}} \cdot \mathcal{Y}_{m_{s}}\right)= & b_{m_{1}} \cdot \mathcal{Y}_{m_{1}} e_{1}+\cdots+b_{m_{s}} \cdot \mathcal{Y}_{m_{s}} e_{s} \\
= & b_{m_{1}} \cdot c_{1} \cdot \frac{\mathcal{Y}_{m_{1}} \cdot \mathcal{X}_{1}}{c_{1} \cdot \mathcal{X}_{1}} e_{1}+\cdots+b_{m_{s}} \cdot c_{s} \cdot \frac{\mathcal{Y}_{m_{s}} \mathcal{X}_{s}}{c_{s} \mathcal{X}_{s}} e_{s} \\
= & b_{m_{1}} \cdot c_{1} \cdot \frac{\mathcal{X}}{\mathcal{X}_{12}}\left(\frac{\mathcal{X}_{12}}{c_{1} \cdot \mathcal{X}_{1}} e_{1}-\frac{\mathcal{X}_{12}}{c_{2} \cdot \mathcal{X}_{2}} e_{2}\right) \\
& +\left(b_{m_{1}} \cdot c_{1}+c_{2} \cdot b_{m_{2}}\right) \cdot \frac{\mathcal{X}}{\mathcal{X}_{23}}\left(\frac{\mathcal{X}_{23}}{c_{2} \cdot \mathcal{X}_{2}} e_{2}-\frac{\mathcal{X}_{23}}{c_{3} \cdot \mathcal{X}_{3}} e_{3}\right) \\
& +\cdots+\left(\sum_{j=1}^{s-1} c_{j} \cdot b_{m_{j}}\right) \cdot \frac{\mathcal{X}}{\mathcal{X}_{s-1 s}}\left(\frac{\mathcal{X}_{s-1 s}}{c_{s-1} \cdot \mathcal{X}_{s-1}} e_{s-1}-\frac{\mathcal{X}_{s-1 s}}{c_{s} \cdot \mathcal{X}_{s}} e_{s}\right) \\
& +\left(\sum_{j=1}^{s} c_{j} \cdot b_{m_{j}}\right) \cdot \frac{\mathcal{X}}{c_{s} \mathcal{X}_{s}} e_{s} \\
= & \sum_{i=1}^{s-1}\left(\sum_{j=1}^{i} c_{j} \cdot b_{m_{j}} \frac{\mathcal{X}}{\mathcal{X}_{i i+1}}\right) S_{i i+1}
\end{aligned}
$$

Definition 8 (Saturation). For power products $\mathcal{X}_{1}, \ldots, \mathcal{X}_{s}$ and a subset $J \subseteq\{1, \ldots, s\}$ we set $\mathcal{X}_{J}=\operatorname{lcm}\left(\mathcal{X}_{j} \mid j \in J\right)$. We say that $J$ is saturated with respect to $\mathcal{X}_{1}, \ldots, \mathcal{X}_{s}$ provided that for all $j \in\{1, \ldots, s\}$ the index $j$ is an element of $J$ if $\mathcal{X}_{j}$ divides $\mathcal{X}_{J}$. We call the subset $J^{\prime} \subseteq\{1, \ldots, s\}$ consisting of all $j$ such that $\mathcal{X}_{j}$ divides $\mathcal{X}_{J}$ the saturation of $J$. We denote with $\operatorname{Sat}\left(\mathcal{X}_{1}, \ldots, \mathcal{X}_{s}\right)$ all saturated subsets of $\{1, \ldots, s\}$ with respect to $\mathcal{X}_{1}, \ldots, \mathcal{X}_{s}$.

Lemma 8. Let $G=\left\{g_{1}, \ldots, g_{s}\right\}$ be a Gröbner basis for an ideal $I$ of $A$ and let $n=\prod_{\ell=1}^{k} p_{\ell}^{e_{\ell}}$ be the prime power decomposition of the characteristic $n$ of $D$. For $1 \leq i \leq s$ let the leading term of $g_{i}$ be denoted by $\operatorname{lt}\left(g_{i}\right)=c_{i} \mathcal{X}_{i}$. If all leading coefficients $c_{i}$ are units in $D$ then for each $1 \leq \ell \leq k$ the set

$$
\begin{aligned}
\mathfrak{B}_{\mathrm{Syz}\left(\operatorname{lt}\left(g_{1}\right), \ldots, \operatorname{lt}\left(g_{s}\right), p_{\ell}^{e_{\ell}}\right)} & =\left\{\left(S_{i j}, 0\right) \mid S_{i j} \in \mathfrak{B}_{\mathrm{Syz}\left(\operatorname{lt}\left(g_{1}\right), \ldots, \operatorname{lt}\left(g_{s}\right)\right)}\right\} \\
& \cup\left\{\frac{p_{\ell}^{e_{\ell}} \mathcal{X}_{J}}{c_{j} \mathcal{X}_{j}} e_{j}-\mathcal{X}_{J} e_{s+1} \mid J \in \operatorname{Sat}\left(\mathcal{X}_{1}, \ldots, \mathcal{X}_{s}\right) \text { and some } j \in J\right\}
\end{aligned}
$$

is a homogeneous generating set for $\operatorname{Syz}\left(\operatorname{lt}\left(g_{1}\right), \ldots, \operatorname{lt}\left(g_{s}\right), p_{\ell}^{e \ell}\right)$.

Proof. Certainly, each element of $\mathfrak{B}_{\mathrm{Syz}\left(\operatorname{lt}\left(g_{1}\right), \ldots, \operatorname{lt}\left(g_{s}\right), p_{\ell}^{e_{\ell}}\right)}$ is vector of $\operatorname{Syz}\left(\operatorname{lt}\left(g_{1}\right), \ldots, \operatorname{lt}\left(g_{s}\right), p_{\ell}^{e_{\ell}}\right)$ as

$$
\left(S_{i j}, 0\right) \cdot\left(\operatorname{lt}\left(g_{1}\right), \ldots, \operatorname{lt}\left(g_{s}\right), p_{\ell}^{e_{\ell}}\right)^{t}=S_{i j} \cdot\left(\operatorname{lt}\left(g_{1}\right), \ldots, \operatorname{lt}\left(g_{s}\right)\right)^{t}=0
$$

and

$$
\frac{p_{\ell}^{e_{\ell}} \mathcal{X}_{J}}{c_{j} \mathcal{X}_{j}} e_{j}-\mathcal{X}_{J} e_{s+1} \cdot\left(\operatorname{lt}\left(g_{1}\right), \ldots, \operatorname{lt}\left(g_{s}\right), p_{\ell}^{e_{\ell}}\right)^{t}=\frac{p_{\ell}^{e_{\ell}} \mathcal{X}_{J}}{c_{j} \mathcal{X}_{j}} \operatorname{lt}\left(g_{j}\right)-\mathcal{X}_{J} \cdot p_{\ell}^{e_{\ell}}=0
$$

for $J \in \operatorname{Sat}\left(\mathcal{X}_{1}, \ldots, \mathcal{X}_{s}\right)$ and some $j \in J$. Furthermore the element $\left(S_{i j}, 0\right)$ is homogeneous of degree $\mathcal{X}_{i j}$ and $\frac{p_{\ell}^{e \ell} \mathcal{X}_{J}}{c_{j} \mathcal{X}_{j}} e_{j}-\mathcal{X}_{J} e_{s+1}$ is a homogeneous syzygy of degree $\mathcal{X}_{j}$. 
Let $h=\left(h_{1}, \ldots, h_{s+1}\right) \in \operatorname{Syz}\left(\operatorname{lt}\left(g_{1}\right), \ldots, \operatorname{lt}\left(g_{s}\right), p_{\ell}^{e \ell}\right)$. If $h_{s+1}=0$ then $\left(h_{1}, \ldots, h_{s}\right)$ is an element of $\operatorname{Syz}\left(\operatorname{lt}\left(g_{1}\right), \ldots, \operatorname{lt}\left(g_{s}\right)\right)$, hence by Lemma $7\left(h_{1}, \ldots, h_{s}, 0\right)$ is finite linear combination of

$$
\left\{\left(S_{i j}, 0\right) \mid S_{i j} \in \mathfrak{B}_{\mathrm{Syz}\left(\mathrm{lt}\left(g_{1}\right), \ldots, \mathrm{lt}\left(g_{s}\right)\right)}\right\} .
$$

Otherwise, using the notation from the proof Lemma 7 , if $h_{s+1} \neq 0$ then for a power product $\mathcal{X}$, the coefficient of $\mathcal{X}$ in the polynomial

$$
h \cdot\left(\operatorname{lt}\left(g_{1}\right), \ldots, \operatorname{lt}\left(g_{s}\right), p_{\ell}^{e_{\ell}}\right)^{t}=\sum_{i=1}^{s} l t\left(g_{i}\right) h_{i}+p_{\ell}^{e_{\ell}} h_{s+1}=\sum_{i=1}^{s} c_{i} \mathcal{X}_{i}\left(\sum_{k=1}^{d_{i}} a_{k}^{(i)} \mathcal{X}_{k}^{(i)}\right)+p_{\ell}^{e_{\ell}}\left(\sum_{k=1}^{d_{s+1}} a_{k}^{(i)} \mathcal{X}_{k}^{(i)}\right)
$$

must be zero for $h_{i}=\sum_{k=1}^{d_{i}} a_{k}^{(i)} \mathcal{X}_{k}^{(i)}$ and $1 \leq i \leq s+1$.

Let

$$
\left\{\mathcal{Y}_{1}, \ldots, \mathcal{Y}_{d}\right\}=\bigcup_{i=1}^{s+1}\left\{\mathcal{X}_{k}^{(i)} \mid 1 \leq k \leq d_{i}\right\}
$$

with $\mathcal{Y}_{1}<\cdots<\mathcal{Y}_{d}$. Then

$$
h_{i}=\sum_{k=1}^{d_{i}} a_{k}^{(i)} \mathcal{X}_{k}^{(i)}=\sum_{\ell=1}^{d} b_{\ell}^{(i)} \mathcal{Y}_{\ell} \text { with }\left\{\begin{array}{ll}
b_{\ell}^{(i)}=a_{k}^{(i)} & \text { if } \mathcal{Y}_{\ell}=\mathcal{X}_{k}^{(i)} \\
b_{\ell}^{(i)}=0 & \text { else }
\end{array} \text { for some index } 1 \leq k \leq d_{i},\right.
$$

for $1 \leq i \leq s+1$.

Furthermore, let $\mathcal{X}$ be a power product and $1 \leq m_{i} \leq d$ be the index such that $\mathcal{X}_{i} \cdot \mathcal{Y}_{m_{i}}=\mathcal{X}$ for $1 \leq i \leq s+1$ with $\mathcal{X}_{s+1}=1$. Then $\left(b_{m_{1}} \mathcal{Y}_{m_{1}}, \ldots, b_{m_{s+1}} \mathcal{Y}_{m_{s+1}}\right)$ is a homogeneous syzygy of degree $\mathcal{X}$. Moreover, we assume that $b_{m_{s+1}} \neq 0$ and consider the index set $J^{\prime}=\left\{j \mid b_{m_{j}} \neq 0\right\} \backslash\{s+1\}$. Let $J \in \operatorname{Sat}\left(\mathcal{X}_{1}, \ldots, \mathcal{X}_{s}\right)$ such that $J^{\prime} \subseteq J$. Then we fix an index $d \in J$ such that $\frac{p_{\ell}^{e} \mathcal{X}_{J}}{c_{d} \mathcal{X}_{d}} e_{d}-\mathcal{X}_{J} e_{s+1} \in$ $\mathfrak{B}_{\mathrm{Syz}\left(\operatorname{lt}\left(g_{1}\right), \ldots, \operatorname{lt}\left(g_{s}\right), p_{\ell}^{e_{\ell}}\right)}$. It follows that

$$
\begin{aligned}
& \left(b_{m_{1}} \mathcal{Y}_{m_{1}}, \ldots, b_{m_{s+1}} \mathcal{Y}_{m_{s+1}}\right)-\left(-b_{m_{s+1}}\right) \cdot\left(\frac{p_{\ell}^{e_{\ell}} \mathcal{X}_{J}}{c_{d} \mathcal{X}_{d}} e_{d}-\mathcal{X}_{J} e_{s+1}\right) \cdot \frac{\mathcal{X}}{\mathcal{X}_{J}} \\
= & \left(b_{m_{1}} \mathcal{Y}_{m_{1}}, \ldots, b_{m_{d}} \mathcal{Y}_{m_{d}}+\frac{b_{m_{s+1}} \cdot p_{\ell}^{e_{\ell}} \mathcal{X}_{J}}{c_{d} \mathcal{X}_{d}} \cdot \frac{\mathcal{X}}{\mathcal{X}_{J}}, b_{m_{d+1}} \mathcal{Y}_{m_{d+1}}, \ldots, b_{m_{s}} \mathcal{Y}_{m_{s}}, b_{m_{s+1}} \mathcal{Y}_{m_{s+1}}-b_{m_{s+1}} \mathcal{X}_{J} \cdot \frac{\mathcal{X}}{\mathcal{X}_{J}}\right) \\
= & \left(b_{m_{1}} \mathcal{Y}_{m_{1}}, \ldots,\left(b_{m_{d}}+\frac{b_{m_{s+1}} \cdot p_{\ell}^{e_{\ell}}}{c_{d}}\right) \mathcal{Y}_{m_{d}}, b_{m_{d+1}} \mathcal{Y}_{m_{d+1}}, \ldots, b_{m_{s}} \mathcal{Y}_{m_{s}}, 0\right)
\end{aligned}
$$

is a homogeneous syzygy with zero in the $(s+1)$-th coordinate and a linear combination of the set $\left\{\left(S_{i j}, 0\right) \mid S_{i j} \in \mathfrak{B}_{\mathrm{Syz}}\left(\mathrm{lt}\left(g_{1}\right), \ldots, \mathrm{lt}\left(g_{s}\right)\right)\right\}$ by Lemma 7 .

Now, we are able to actually prove Lemma 6 from Section 4.2.

Proof (Lemma 6). By Theorem 6 the set $G_{\ell}=\left\{p_{\ell}^{e_{\ell}}, g_{1}, \ldots, g_{s}\right\}$ is a Gröbner basis for for the ideal $J_{i}=\left\langle p_{\ell}^{e_{\ell}}, g_{1}, \ldots, g_{s}\right\rangle$ if and only if for each element $h \in \mathfrak{B}_{\operatorname{Syz}\left(\operatorname{lt}\left(g_{1}\right), \ldots, \operatorname{lt}\left(g_{s}\right), p_{\ell}^{e_{\ell}}\right)}$ the relation $h \cdot\left(g_{1}, \ldots, g_{s}, p_{l}^{e_{l}}\right)^{t} \stackrel{G_{l}}{\longrightarrow}+0$ holds.

Let $h \in \mathfrak{B}_{\mathrm{Syz}}\left(\operatorname{lt}\left(g_{1}\right), \ldots, \operatorname{lt}\left(g_{s}\right), p_{l}^{e_{l}}\right)$, then by Lemma 8 either $h$ is an element of

$$
\left\{\left(S_{i j}, 0\right) \mid S_{i j} \in \mathfrak{B}_{\mathrm{Syz}\left(\operatorname{lt}\left(g_{1}\right), \ldots, \operatorname{lt}\left(g_{s}\right)\right)}\right\}
$$


or $h$ is an element of

$$
\left\{\frac{p_{\ell}^{e_{\ell}} \mathcal{X}_{J}}{\operatorname{lt}\left(g_{j}\right)} e_{j}-\mathcal{X}_{J} e_{s+1} \mid J \in \operatorname{Sat}\left(\operatorname{lp}\left(g_{1}\right), \ldots, \operatorname{lp}\left(g_{s}\right)\right) \text { and some } j \in J\right\} .
$$

For the first case, we observe that

$$
h \cdot\left(g_{1}, \ldots, g_{s}, p_{l}^{e_{l}}\right)^{t}=\sum_{i=1}^{s} h_{i} g_{i}=\left(h_{1}, \ldots, h_{s}\right) \cdot\left(g_{1}, \ldots, g_{s}\right)^{t}
$$

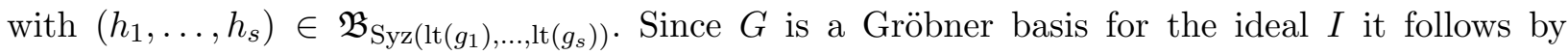
Theorem 6 that $h \cdot\left(g_{1}, \ldots, g_{s}, p_{\ell}^{e_{\ell}}\right)^{t} \stackrel{G}{\longrightarrow} 0$ and thus $\left(g_{1}, \ldots, g_{s}, p_{\ell}^{e_{\ell}}\right)^{t} \stackrel{G_{\ell}}{\longrightarrow} 0$.

In the other case, we have

$$
h=\frac{p_{\ell}^{e_{\ell}} \mathcal{X}_{J}}{\operatorname{lt}\left(g_{j}\right)} e_{j}-\mathcal{X}_{J} e_{s+1}
$$

for some $J \in \operatorname{Sat}\left(\operatorname{lp}\left(g_{1}\right), \ldots, \operatorname{lp}\left(g_{s}\right)\right)$ and some $j \in J$. Furthermore,

$$
h \cdot\left(g_{1}, \ldots, g_{s}, p_{\ell}^{e_{\ell}}\right)^{t}=\frac{p_{\ell}^{e_{\ell}} \mathcal{X}_{J}}{\operatorname{lt}\left(g_{j}\right)} g_{j}-p_{\ell}^{e_{\ell}} \mathcal{X}_{J}=\frac{p_{\ell}^{e_{\ell}} \mathcal{X}_{J}}{\operatorname{lt}\left(g_{j}\right)}\left(\operatorname{lt}\left(g_{j}\right)+g^{\prime}\right)-p_{\ell}^{e_{\ell}} \mathcal{X}_{J}=\frac{p_{\ell}^{e_{\ell}} \mathcal{X}_{J}}{\operatorname{lt}\left(g_{j}\right)} g^{\prime}=\frac{g^{\prime} \cdot \mathcal{X}_{J}}{\operatorname{lt}\left(g_{j}\right)} p_{\ell}^{e_{\ell}}
$$

for $g_{j}=\operatorname{lt}\left(g_{j}\right)+g^{\prime}$, thus $h \cdot\left(g_{1}, \ldots, g_{s}, p_{\ell}^{e_{\ell}}\right)^{t} \stackrel{\left\{p_{\ell}^{e_{\ell}}\right.}{\longrightarrow}+0$ implying that $h \cdot\left(g_{1}, \ldots, g_{s}, p_{\ell}^{e_{\ell}}\right)^{t} \stackrel{G_{\ell}}{\longrightarrow} 0$. 